\title{
An Approach to $\mathcal{N}=4 A D E$ Gauge Theory on $K 3$
}

\author{
Masao Jinzenji ${ }^{\dagger}$, Toru Sasaki* \\ ${ }^{\dagger}$ Division of Mathematics, Graduate School of Science, Hokkaido University, Sapporo \\ 060-0810, Japan \\ *Department of Physics, Hokkaido University, Sapporo 060-0810, Japan \\ ${ }^{\dagger}$ jin@math.sci.hokudai.ac.jp \\ *sasaki@particle.sci.hokudai.ac.jp
}

\begin{abstract}
We propose a recipe for determination of the partition function of $\mathcal{N}=4 A D E$ gauge theory on $K 3$ by generalizing our previous results of the $S U(N)$ case. The resulting partition function satisfies Montonen-Olive duality for $A D E$ gauge group.
\end{abstract}




\section{Introduction}

This article is the continuation of our previous works [6, 7]. Based on the understanding obtained in these works, we try to determine the partition function of $\mathcal{N}=4 A D E$ gauge theory on $K 3$.

$\mathcal{N}=4$ theory is one of the good laboratories of testing duality conjecture. It is well-known that $\mathcal{N}=4$ theory has the largest possible number of super-symmetry for a four-dimensional theory without gravity. Furthermore, it is believed to be exactly finite and conformally invariant. The duality of this theory is believed to be the one proposed by Montonen and Olive. On the basis of this thought, the study of $\mathcal{N}=4 S U(N)$ gauge theory has enjoyed many progresses with the aid of the $S$-duality conjecture: the sharpened version of Montonen-Olive duality [21, 11, 13]. On the other hand, there has been almost no attempt to determine the partition function of $D, E$ gauge theory in spite of the fact that the $S$ duality conjecture for these cases has already been established [22, 16].

In our previous work, we have studied $\mathcal{N}=4$ super-symmetric Yang-Mills theory on orbifold- $T^{4} / \mathbf{Z}_{2}$ for $S U(2)$ [6] and for $S U(N)$ [7]. In these works, we tried to realize the following geometrical picture at the partition function level. A special class of $K 3$ surfaces can be constructed from blowing up sixteen singularities of the quotient space $T^{4} / \mathbf{Z}_{2}=S_{0}$. Therefore, the partition function on $K 3$ should be given as the product of the partition function of $S_{0}$ and the blow-up formula [24, 21, 9]. This attempt was successful not only for $S U(2)$ but also for $S U(N)$. The verification of this attempt mainly depends on the following two facts. The first one is that the $S U(N)$ partition function on $K 3$ can be described by a Hecke transformation of order $N$ of $1 / \eta(\tau)^{24}[21,13,26$, 11]. The second one is that $1 / \eta(\tau / N)$ can be written as the form $\theta_{A_{N-1}}^{2}(\tau) / \eta(\tau)^{N}$, where $\theta_{A_{N-1}}^{2}(\tau)$ is a kind of $A_{N-1}$ theta function [7]. This identity is easily verified from the celebrated denominator identity of the affine Lie algebra [8, 12]. These two facts enabled us to rewrite the partition function of $S U(N)$ theory on $K 3$ into the desired form.

Looking back at the above verification, we can speculate that inversion of the above process can be used to guess the form of the partition function of general $A D E$ gauge theory. Fortunately, we already have the works on $A D E$ blow-up formulas [24, 19, 9], which states that principal part of $A D E$ blow-up formulas have the form of $A D E$ theta functions $\theta_{A, D, E}(\tau)$. In this paper, we proceed further and assert that the partition function of $A D E$ gauge theory should take the form of $\left(\theta_{A D E}(\tau) / \eta(\tau)^{r+1}\right)^{24}(r$ is the rank of the gauge group). This form can be verified by using the fact that $\mathcal{N}=4 A D E$ gauge theory on $K 3$ is obtained from Type II A string theory compactified on $K 3 \times T^{2} \times(A L E$ space). By plugging this assertion into the denominator identity, we can see that the $D, E$ partition functions can be rewritten as the form of $(\eta \text { - product })^{24}$. As was pointed out before, the partition function of $S U(N)$ gauge theory on $K 3$ surface is written only by $\eta$ function. This is because the moduli space of rank $N$ semi-stable sheaves is described by Hilbert scheme of $K 3$ surface itself [17, 18, 26]. Physical interpretation of this fact is also given from the point of view of Type IIA-Heterotic duality. Though we have not obtain analogous results on the moduli space of $D, E$ vector bundles, we already have many beautiful results on $K 3$ surface, which is enough for us to expect that the partition function of $D, E$ gauge theory should be written only by $\eta$-product. Therefore, we use the above $(\eta \text {-product })^{24}$ as the primary building block of the $D, E$ partition function on $K 3$. Next step of our construction is found from the analogy with the $A_{N-1}$ case. The corresponding primary 
building block of the $A_{N-1}$ partition function is $1 / \eta(\tau / N)^{24}$. Then we take $S L(2, Z)$ orbit of this function (precisely speaking, we have to include the transformation $\tau \rightarrow \tau+1 / m$ in some cases). After that, we take linear combination of all the building blocks so obtained, whose coefficients are determined to satisfy the $S$-duality conjecture, as was done in VafaWitten 's original work 21]. The result is nothing but the order $N$ Hecke transformation of $1 / \eta(\tau)^{24}$. Hence we applied the same procedure by taking ( $\eta$-product) $)^{24}$ as the primary function. As a result, we found that the above procedure also works quite well in this case. Remarkably enough, the resulting partition function completely satisfies Montonen-Olive duality! 16].

Unfortunately, our results are not complete from the geometrical point of view. It is well-known that the partition function is the generating function of the Euler number of the moduli space of irreducible anti-self-dual connection. For $A D E$ gauge group, we have Atiyah-Hitchin-Singer formula that gives us the dimension of the moduli space of irreducible anti-self-dual connection [1]. This formula tells us that if the instanton number $k$ is less than $\operatorname{rank}\left(\mathcal{G}_{A D E}\right)+1$, the moduli space is empty. On the other hand, the partition function that we have derived has non-vanishing coefficients in the region $k \leq r$. Up to now, we think that these phenomena is caused by existence of reducible connections. Especially in the case of $S U(N)$ theory on the manifold $b_{2}^{+} \leq 1$, it is well-known that existence of reducible connections gives rise to the appearance of the holomorphic anomaly in the partition function. Thus we speculate that the true partition function of $D, E$ theory on $K 3$ should have the holomorphic anomaly. In other words, we have derived the holomorphic part of the partition function of $D, E$ gauge theory. Therefore, we should search for an analogue of the holomorphic anomaly equation used in 13] as the second step. This problem will be discussed in our subsequent paper.

The organization of this paper is the following: In Sec.2 we review the Vafa-Witten theory in the $S U(N)$ case and discuss generalization to the $D, E$ gauge theory. Next, we look carefully at the detailed structure of the duality conjecture on the partition function of $S U(N)$ theory as warming-ups. In Sec.3 we estimate the form of the $D, E$ partition functions on $K 3$ from the point of view of Type II A string theory compactified on $K 3 \times$ $T^{2} \times(A L E$ space). In Sec.4 we combine the above result with the denominator identity of affine Lie algebra and determine the $D, E$ partition function on $K 3$ up to some small rank. In Sec.5 we point out that our partition function of $D, E$ gauge theory on $K 3$ does not satisfy the gap condition and discuss the possible improvement parallel to the treatment

of holomorphic anomaly in the $\frac{1}{2} K 3$ case. In Sec. 6 we conclude and discuss the remaining problems.

\section{Vafa-Witten Theory}

\section{1 't Hooft Flux for ADE Gauge Theory}

In this subsection, we introduce the 't Hooft flux for $A D E$ gauge theory. To this aim, we first consider the non-Abelian version of the Dirac quantization condition [16]:

$$
\exp \left(4 \pi i e F_{0}\right)=1,
$$

where $F_{0}$ is a Lie algebra valued matrix of $A D E$ gauge group $\mathcal{G}$ and appears in the solution of the magnetic monopole equation. By using gauge transformation, $F_{0}$ can be rewritten 
into the form:

$$
e F_{0}=\sum_{j=1}^{\operatorname{rank}(\mathcal{G})} \beta_{j} T_{j},
$$

where the $T_{j}$ 's are any appropriately normalized set of mutually commutative generators of $\mathcal{G}$. Here we introduce the root lattice $\Lambda(\mathcal{G})$. Then (2.1) turns out to be

$$
2 \beta \cdot \alpha \in \mathbf{Z} \text {, for all } \alpha \in \Lambda(\mathcal{G}) \text {. }
$$

This condition tells us that $\beta$ should belong to the dual lattice (or weight lattice) $\Lambda\left(\mathcal{G}^{\vee}\right)$. On the other hand, it is well-known that

$$
\Lambda\left(\mathcal{G}^{\vee}\right) / \Lambda(\mathcal{G}) \cong \Gamma_{\mathcal{G}}
$$

where $\Gamma_{\mathcal{G}}$ is center of $\mathcal{G}$. In this way, Montonen and Olive proposed that $A D E$ gauge group $\mathcal{G}$ is dual to $\mathcal{G} / \Gamma_{\mathcal{G}}$. From this proposal, it is natural to assume that 't Hooft flux of the dual group $\mathcal{G} / \Gamma_{\mathcal{G}}$ is given by $v \in H^{2}\left(X, \Gamma_{\mathcal{G}}\right)$. For later use, we write down $\Gamma_{\mathcal{G}}$ in the following table:

\begin{tabular}{|c|c|}
\hline $\mathcal{G}$ & $\Gamma_{\mathcal{G}}$ \\
\hline$A_{N-1}$ & $\mathbf{Z}_{N}$ \\
\hline$D_{2 N}$ & $\mathbf{Z}_{2} \times \mathbf{Z}_{2}$ \\
\hline$D_{2 N+1}$ & $\mathbf{Z}_{4}$ \\
\hline$E_{6}$ & $\mathbf{Z}_{3}$ \\
\hline$E_{7}$ & $\mathbf{Z}_{2}$ \\
\hline$E_{8}$ & trivial \\
\hline
\end{tabular}

Classification of the type of the 't Hooft flux of $A_{N-1}(N$ : prime) is already studied [21, 11]. However, general $N$ case is more complicated. In Sec.2.3, we will show explicitly how $v \in H^{2}\left(K 3, \Gamma_{\mathcal{G}}\right)$ are classified in the case of general $A D E$ gauge group.

\subsection{Vafa-Witten Conjecture for ADE Gauge Theory}

Following [21, 11, 25], we review the general structure of Vafa-Witten conjecture. Twisted $\mathcal{N}=4$ gauge theory on the manifold with suitable vanishing theorem causes remarkable simplification [21]. That is, its partition function has the form of the summation of the Euler number of the moduli space of the ASD equations. More precisely, for twisted $\mathcal{N}=4$ $\mathcal{G} / \Gamma_{\mathcal{G}}$ gauge theory with 't Hooft flux $v \in H^{2}\left(X, \Gamma_{\mathcal{G}}\right)$ on $X$, we propose that the partition function of this theory is given by the formula:

$$
Z_{v}^{X}(\tau):=q^{-\frac{(r+1) \chi(X)}{24}} \sum_{k} \chi(\mathcal{M}(v, k)) q^{k} \quad(q:=\exp (2 \pi i \tau)),
$$

where $\mathcal{M}(v, k)$ is the moduli space of $\mathrm{ASD}$ connections associated to $\mathcal{G} / \Gamma_{\mathcal{G}}$-principal bundle with 't Hooft flux $v$ and fractional instanton number $k \in \frac{1}{2\left|\Gamma_{\mathcal{G}}\right|} \mathbf{Z}$. In (2.5), $\tau$ is the gauge coupling constant including theta angle, $\chi(X)$ is Euler number of $X$ and $r$ is the rank 
of $\mathcal{G}$. Introduction of $q^{-\frac{1}{24}}$ factor is required by the modular property like the case of $\eta(\tau)$ function. For $S U(N) / \mathbf{Z}_{N}$, this conjecture is nothing but Vafa-Witten's [21]. With this result, Vafa and Witten conjectured the behavior of the partition functions under the action of $S L(2, \mathbf{Z})$ on $\tau$. They started with 't Hooft's work [20] in mind. In [20], the path integral with $\mathbf{Z}_{N}$-valued electric flux and that with magnetic flux is related by Fourier transform. Vafa and Witten combined the conjecture of strong/week duality to this 't Hooft's result. Their conjecture is summarized by the following formula:

$$
Z_{v}^{X}\left(-\frac{1}{\tau}\right)=\left|\Gamma_{\mathcal{G}}\right|^{-\frac{b_{2}(X)}{2}}\left(\frac{\tau}{i}\right)^{-\frac{\chi(X)}{2}} \cdot \sum_{u \in H^{2}\left(X, \Gamma_{\mathcal{G}}\right)} \zeta_{\mathcal{G}}^{u \cdot v} Z_{u}^{X}(\tau)
$$

where $\zeta_{\mathcal{G}}=\exp \left(\frac{2 \pi i}{N_{\mathcal{G}}}\right)$. We conjecture that this duality relation is also valid for the general $A D E$ partition function (2.5).

For later use, we introduce the notation:

$$
\begin{aligned}
Z_{\mathcal{G}}^{X}(\tau) & :=\frac{1}{\left|\Gamma_{\mathcal{G}}\right|} Z_{t}^{X}(\tau), \\
Z_{\mathcal{G} / \Gamma_{\mathcal{G}}}^{X}(\tau) & :=\sum_{u \in H^{2}\left(X, \Gamma_{\mathcal{G}}\right)} Z_{u}^{X}(\tau) .
\end{aligned}
$$

In this notation, we can obtain the following formula from (2.6):

$$
Z_{t}^{X}\left(-\frac{1}{\tau}\right)=\left|\Gamma_{\mathcal{G}}\right|^{-\frac{b_{2}(X)}{2}}\left(\frac{\tau}{i}\right)^{-\frac{\chi(X)}{2}} Z_{\mathcal{G} / \Gamma_{\mathcal{G}}}^{X}(\tau)
$$

This formula is one of the key points on their explicit determination of the form of the partition function of complex surface with ample canonical bundle.

\subsection{Counting the Number of Orbits on $K 3$}

In the rest of this paper, we will concentrate on $A D E$ gauge theory on $K 3$.

In [21], Vafa and Witten classified the types of $S O(3)=S U(2) / \mathbf{Z}_{2}$ theory with 't Hooft flux $v \in H^{2}\left(K 3, \mathbf{Z}_{2}\right)$ on $K 3$. Note that $b^{2}(K 3)=22$, and $v$ can takes $2^{22}$ values. They used the property that diffeomorphism invariant of $v$ is given by $v^{2} \bmod 4(v \neq 0)$ and $v=0$. Especially on $K 3$ there are only three types of partition functions. These three types are called as even $\left(v^{2} \equiv 0 \bmod 4\right.$ and $\left.v \neq 0\right)$, odd $\left(v^{2} \equiv 2 \bmod 4\right)$ and trivial $(v=0)$ respectively. Furthermore numbers of each type are denoted as $n_{\text {even }}, n_{\text {odd }}$ and $n_{0}$ respectively. These numbers are concretely counted in [21]. Of course they sum up to $n_{0}+n_{\text {even }}+n_{\text {odd }}=2^{22}$. It is well known that the intersection form on $H^{2}(K 3, \mathbf{Z})$ is $H^{\oplus 3} \oplus\left(-E_{8}\right)^{\oplus 2}$. However Vafa and Witten took $H^{\oplus 11}$ (the direct sum of 11 copies of $H$ ) instead of $H^{\oplus 3} \oplus\left(-E_{8}\right)^{\oplus 2}$ itself as the intersection form on $H^{2}(K 3, \mathbf{Z})$ for convenience. Here

$$
H=\left(\begin{array}{ll}
0 & 1 \\
1 & 0
\end{array}\right) \text {. }
$$

This replacement makes counting the numbers of orbits very simple. We follow this replacement in this paper. From now on, we generalize the above result of the $S U(2)$ case 
to the case of general $A D E$ group. First we count naive number of orbits for $S U(N) / \mathbf{Z}_{N}$. We denote 't Hooft flux for $S U(N) / \mathbf{Z}_{N}$ by,

$$
v_{N} \in\left(H^{\oplus 11}\right) \otimes_{\mathbf{Z}} \mathbf{Z}_{N}=: H_{N} .
$$

Roughly speaking, the word "naive" means that $v_{N}$ has no restriction. Explicitly, the naive number of orbits of $S U(N) / \mathbf{Z}_{N}$ is defined as follows:

$$
n_{j}(N)=\text { number of } v_{N}^{2} \equiv 2 j \bmod 2 N .
$$

We introduce $V$ whose intersection form is given by $H$. For $V$, we define,

$$
l_{j}=\text { numbers of } V^{2} \equiv 2 j \bmod 2 N .
$$

With this definition, one can build the recursive formula for computing the number of orbits on the manifold with $H^{\oplus n}$. The solution for $n=11$ case is

$$
n_{j}(N)=\frac{1}{N} \sum_{k=0}^{N-1} m_{k}^{11} \zeta_{N}^{j k},
$$

where

$$
m_{k}=\sum_{j=0}^{N-1} l_{j} \zeta_{N}^{j k}
$$

and

$$
\zeta_{N}=\exp \left(\frac{2 \pi i}{N}\right)
$$

From now on, we introduce a subscript " $t$ " such as $n_{t}$, which corresponds to the trivial flux $v=0$.

$A_{N-1}: N$ prime

In this part, we count the number of orbits of $S U(N) / \mathbf{Z}_{N}: N$ prime. If $N$ is prime number, one finds $m_{0}=2 N-1, m_{j}=N,(j=1, \ldots, N-1)$. With some algebra, one can find

$$
\begin{aligned}
n_{0}^{N} & =\text { number of } v_{N}^{2} \equiv 0 \bmod 2 N \text { and } v_{N} \neq 0 \\
& =n_{0}(N)-1=N^{21}+(N-1) N^{10}-1, \\
n_{j}^{N} & =\text { number of } v_{N}^{2} \equiv 2 j \bmod 2 N \\
& =n_{j}(N)=N^{21}-N^{10}, j=1, \ldots, N-1, \\
n_{t}^{1} & =\text { number of } v_{N}=0 \\
& =n_{t}=1 .
\end{aligned}
$$

\section{$A_{N-1}: N$ non-prime}

In this part, we count the number of orbits of $S U(N) / \mathbf{Z}_{N}: N$ non-prime. That is $N=$ $p_{1}^{m_{1}} p_{2}^{m_{2}} \cdots p_{l}^{m_{l}}$ case $\left(p_{j}\right.$ is a prime). Here we took the following two cases as typical examples: (i) $N=p^{m}$, (ii) $N=p q$. Other cases are straightforward generalization of these two cases. Before moving to specific discussion, we explain general structure of non-prime $N$ 
case. For non-prime $N$, there are several blocks of type, which are classified by all possible divisor of $N=p_{1}^{m_{1}} p_{2}^{m_{2}} \cdots p_{l}^{m_{l}}$. That is

$$
p_{1}^{M_{1}} p_{2}^{M_{2}} \cdots p_{l}^{M_{l}}, 0 \leq M_{1} \leq m_{1}, 0 \leq M_{2} \leq m_{2}, \ldots, 0 \leq M_{l} \leq m_{l} .
$$

Each block labeled by $p_{1}^{M_{1}} p_{2}^{M_{2}} \cdots p_{l}^{M_{l}}$ has the types $j\left(0 \leq j \leq p_{1}^{M_{1}} p_{2}^{M_{2}} \cdots p_{l}^{M_{l}}-1\right)$. Thus we denote the number of orbits of block $m$ and type $j$ by $n_{j}^{m}$. Now, we give explicit

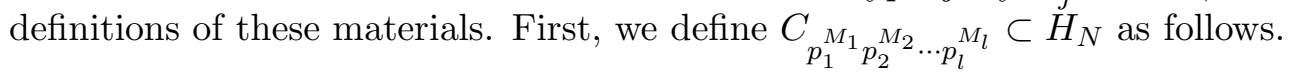

$$
C_{p_{1}^{M_{1}} p_{2}^{M_{2}} \ldots p_{l}^{M_{l}}}:=\left\{v_{N} \in H_{N} \mid p_{1}^{M_{1}} p_{2}^{M_{2}} \cdots p_{l}^{M_{l}} v_{N}=0\right\}
$$

Notice that $C_{m} \subset C_{n}$ if and only if $m \mid n$, and we can easily see the following equality:

$$
C_{i_{1}} \cap C_{i_{2}} \cap C_{i_{3}} \cap \cdots \cap C_{i_{j}}=C_{\operatorname{gcd}\left(i_{1}, i_{2}, \cdots, i_{j}\right)} .
$$

Since the condition $p_{1}^{M_{1}} p_{2}^{M_{2}} \cdots p_{l}^{M_{l}} v_{N}=0$ means that all the components of $v_{N}$ must be multipliers of $p_{1}^{m_{1}-M_{1}} p_{2}^{m_{2}-M_{2}} \cdots p_{l}^{m_{l}-M_{l}}$, we can construct a natural isomorphism:

$$
\begin{aligned}
& \phi_{p_{1} M_{1}} p_{2}^{M_{2} \ldots p_{l}^{M_{l}}}: C_{p_{1}^{M_{1}} p_{2}^{M_{2}} \cdots p_{l}^{M_{l}}} \rightarrow H_{p_{1}^{M_{1}} p_{2}^{M_{2}} \cdots p_{l}^{M_{l}}}, \\
& \phi\left(v_{N}\right):=\frac{1}{p_{1}^{m_{1}-M_{1}} p_{2}^{m_{2}-M_{2}} \cdots p_{l}^{m_{l}-M_{l}}} v_{N} \in H_{p_{1}^{M_{1}} p_{2}^{M_{2}} \cdots p_{l}^{M_{l}}} .
\end{aligned}
$$

With these set-up, we define the block $B_{p_{1}^{M_{1}}} p_{2}^{M_{2} \ldots p_{l} M_{l}}$ as follows:

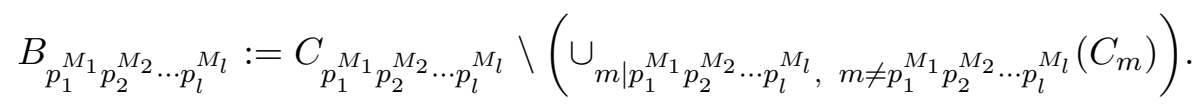

Then the number $n_{j}^{p_{1}^{M_{1}} p_{2}^{M_{2}} \cdots p_{l}^{M_{l}}}$ is nothing but the number of $v_{N} \in B_{p_{1}^{M_{1}} p_{2}^{M_{2}} \ldots p_{l}^{M_{l}}}$ that satisfies the condition:

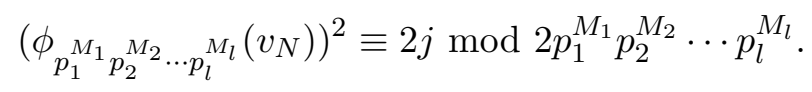

(i) $N=p^{m}$ case

In this case, we have $m$ blocks $B_{p^{M}}=C_{p^{M}} \backslash C_{p^{M-1}}(M=0,1,2, \cdots, m)$ and each block $B_{p^{M}}$ has $p^{M}$ types. Notice that we have the following inclusion sequence in this case:

$$
\{0\}=C_{1} \subset C_{p} \subset C_{p^{2}} \subset \cdots \subset C_{p^{m-1}} \subset C_{p^{m}}=H_{p^{m}} .
$$

Since we have the isomorphism $\phi_{p^{M}}: C_{p^{M}} \rightarrow H_{p^{M}}$, we can write down $n_{j}^{p^{M}}$ in terms of naive numbers $n_{i}\left(p^{M}\right)$ and $n_{l}\left(p^{M-1}\right)$. The result is the following:

Block $B_{p^{M}},(M=m, m-1, \ldots, 1)$

$$
n_{k p^{2}}^{p^{M}}=n_{k p^{2}}\left(p^{M}\right)-\sum_{l=0}^{p-1} n_{l p^{M-2}+k}\left(p^{M-1}\right), k=0, \ldots, p^{M-2}-1,
$$




$$
n_{j}^{p^{M}}=n_{j}\left(p^{M}\right), j=1, \ldots, p^{M}-1 \text { and } j \neq k p^{M-2},
$$

Block $B_{1}$,

$$
n_{t}^{1}=n_{t}=1
$$

For later use, we explicitly write down the results in the $N=4$ case.

$N=4$ case

$$
\begin{aligned}
n_{0}^{4} & =n_{0}(4)-n_{0}(2)-n_{1}(2)=2^{42}+2^{31}-2^{21} \\
n_{1}^{4} & =n_{1}(4)=2^{42}-2^{31} \\
n_{2}^{4} & =n_{2}(4)=2^{42}+2^{31}-2^{21}, \\
n_{3}^{4} & =n_{3}(4)=2^{42}-2^{31} \\
n_{0}^{2} & =n_{0}(2)-n_{t}=2^{21}+2^{10}-1, \\
n_{1}^{2} & =n_{1}(2)=2^{21}-2^{10}, \\
n_{t}^{1} & =n_{t}=1 .
\end{aligned}
$$

(ii) $N=p q$ case

In this case, we have 4 blocks:

$$
B_{p q}=C_{p q} \backslash\left(C_{p} \cup C_{q}\right), B_{p}=C_{p} \backslash C_{1}, B_{q}=C_{q} \backslash C_{1}, B_{1}=C_{1}=\{0\}, \quad\left(C_{p} \cap C_{q}=C_{1}\right) .
$$

Therefore, we can evaluate $n_{j}^{m}$ by use of naive numbers in the same way as the previous case.

Block $B_{p q}$,

$$
\begin{gathered}
n_{0}^{p q}=n_{0}(p q)-n_{0}(q)-n_{0}(p)+n_{t}, \\
n_{k p}^{p q}=n_{k p}(p q)-n_{l_{k}^{q}}(q), \quad k=0, \ldots, q-1, \\
n_{k q}^{p q}=n_{k q}(p q)-n_{l_{k}^{p}}(p), \quad k=0, \ldots, p-1, \\
n_{j}^{p q}=n_{j}(p q), \quad j=1, \ldots, p q-1, \quad j \neq k p, \quad j \neq k q,
\end{gathered}
$$

where $l_{k}^{q}$ and $l_{k}^{p}$ satisfy

$$
l_{k}^{q} p^{2} \equiv 2 k p \bmod 2 p q, \quad l_{k}^{p} q^{2} \equiv 2 k q \bmod 2 p q .
$$

Block $B_{p}$,

$$
\begin{gathered}
n_{0}^{p}=n_{0}(p)-n_{t}, \\
n_{j}^{p}=n_{j}(p), \quad j=1, \ldots, q-1,
\end{gathered}
$$

Block $B_{q}$, numbers are given by $p \leftrightarrow q$.

Block $B_{1}$,

$$
n_{t}^{1}=n_{t}=1 .
$$

In the rest of this subsection, we count the number of orbits of $\mathcal{G} / \Gamma_{\mathcal{G}}$. However the counting is the same as $S U(N) / \mathbf{Z}_{N}$ case except for $D_{2 N} / \mathbf{Z}_{2} \times \mathbf{Z}_{2}$. 
$D_{2 N+1} / \mathbf{Z}_{4}, E_{6} / \mathbf{Z}_{3}$ and $E_{7} / \mathbf{Z}_{2}$

The counting of $D_{2 N+1} / \mathbf{Z}_{\mathbf{4}}, E_{6} / \mathbf{Z}_{3}$ and $E_{7} / \mathbf{Z}_{2}$ cases is the same as those of $S U(4) / \mathbf{Z}_{4}$, $S U(3) / \mathbf{Z}_{3}$ and $S U(2) / \mathbf{Z}_{2}$ cases respectively, because our counting only depends on the center $\Gamma_{\mathcal{G}}$.

$E_{8}$

$E_{8}$ has only one orbit and the result is

$$
n_{t}^{1}=1
$$

This result is just a reflection of the well-known fact that $E_{8}$ lattice is self-dual.

$D_{2 N} / \mathbf{Z}_{2} \times \mathbf{Z}_{2}$

$D_{2 N} / \mathbf{Z}_{2} \times \mathbf{Z}_{2}$ must be classified by $v^{2} \bmod 2$, because $v^{2}$ takes value on $\mathbf{Z}_{2}$. This classification produces degeneration $\mathbf{Z}_{2} \times \mathbf{Z}_{2} \rightarrow \mathbf{Z}_{2} \quad((m, n) \rightarrow m+n)$. The correct counting including this degeneration is given by the following treatment. We regard the counting problem in this case as the one of $S U(2) / \mathbf{Z}_{2}$ theory on a manifold with the replaced intersection form $H^{\oplus 11} \rightarrow H^{\oplus 22}$. This treatment reproduces the desired degeneration and the correct numbers. The result is

$$
\begin{aligned}
& n_{0}^{2}=2^{43}+2^{21}-1 \\
& n_{1}^{2}=2^{43}-2^{21} \\
& n_{t}^{1}=1
\end{aligned}
$$

\subsection{Partition Function for $S U(N)$ Gauge Theory on $K 3$}

Following [21, 11, 13], we derive the $S U(N)$ partition function on $K 3$ as a reconstruction of [21, 11, 13. By using the picture that $\mathcal{N}=4 U(1)$ gauge theory is given by single $M 5$ brane wrapped around $K 3 \times T^{2}$, one can understand that $U(1)$ partition function on $K 3$ is given by

$$
\frac{1}{\eta(\tau)^{24}}=: G(\tau)
$$

Here we ignore the contribution from the $U(1)$ fluxes.

To obtain $\mathcal{N}=4 U(N)$ gauge theory on $K 3$, we consider $N$ coincident $M 5$ brane wrapped around $K 3 \times T^{2}$. Furthermore by fixing 't Hooft flux trivial $v=0$, we obtain $\mathcal{N}=4 S U(N)$ gauge theory on $K 3$. In this picture, Hecke transformation naturally appears as the effect of summing up all the ways of wrapping $T^{2}$ in $K 3 \times T^{2} N$ times by $T^{2}$ in M5 brane. However in the remaining subsection, we apply a more mechanical method for considering the generalization to $D, E$ gauge theory. Of course the result is the same as the result from the above picture.

First we consider the primary function

$$
G_{0}(\tau):=\left(\frac{1}{\eta\left(\frac{\tau}{N}\right)}\right)^{24}
$$


as a piece of $Z_{S U(N)}(\tau)$. Next, by transforming $G_{0}(\tau)$ by $S L(2, \mathbf{Z})$, we obtain the other pieces of $Z_{S U(N)}(\tau)$. Following [21], we introduce some combination of the above functions $Z_{j}(\tau)$ corresponding to the $S U(N) / \mathbf{Z}_{N}$ partition function with $v_{N}^{2} \equiv 2 j \bmod 2 N$. By requiring Montonen-Olive duality (2.8) between $Z_{t}(\tau)$ and $Z_{S U(N) / \mathbf{Z}_{N}}$, we can determine the coefficients of $G$ 's in $Z_{j}(\tau)$.

Let us move to writing out the concrete $S U(N)$ partition function for three cases, prime $N$, non-prime $N=p^{m}$ and $N=p q$.

$A_{N-1}: N$ prime By transforming $G_{0}(\tau)$ by $S L(2, \mathbf{Z})$, we obtain $N+1$ functions,

$$
\begin{gathered}
G_{j}(\tau):=\left(\frac{1}{\eta\left(\frac{\tau+j}{N}\right)}\right)^{24}, j=0, \ldots, N-1, \\
H_{0}(\tau):=\left(\frac{1}{\eta(N \tau)}\right)^{24} .
\end{gathered}
$$

Following [21], we define the following partition functions,

$$
\begin{gathered}
Z_{j}(\tau):=\frac{1}{N}\left(G_{0}(\tau)+\zeta_{N}^{-j} G_{1}(\tau)+\cdots+\zeta_{N}^{-j(N-1)} G_{N-1}(\tau)\right), j=0, \ldots, N-1, \\
Z_{t}(\tau):=\frac{1}{N^{2}} H_{0}(\tau)+Z_{0}(\tau),
\end{gathered}
$$

From these partition functions and the numbers in Sec.2.3, we obtain

$$
Z_{S U(N) / \mathbf{Z}_{N}}(\tau):=\sum_{v} Z_{v}(\tau)=n_{0} Z_{0}(\tau)+\cdots+n_{N-1} Z_{N-1}(\tau)+n_{t} Z_{t}(\tau) .
$$

(2.44) and (2.45) satisfy the Montonen-Olive duality (2.6). We will call the functions appeared in the partition functions as $G$ functions. Most of the $G$ functions are obtained from $S L(2, \mathbf{Z})$ transformation of $G_{0}(\tau)$, but some $G$ functions must be added such as in the following non-prime $N$ cases.

Determination of the partition function for non-prime $N$ case has the same structure as that of the counting the number of orbits. The result is the following.

\section{$A_{N-1}: N$ non-prime}

(i) $N=p^{m}$ case

We introduce the following $G$ functions of block $p^{M}$ and type $j$ :

Block $p^{M},(M=m, m-1, \ldots, 0)$,

$$
G_{j}^{p^{M}}(\tau):=\left(\frac{1}{\eta\left(\frac{p^{m-M} \tau+j}{p^{M}}\right)}\right)^{24}, j=0, \ldots, p^{M}-1 .
$$

Note that most of these functions are obtained by $S L(2, \mathbf{Z})$ transformation from $G_{0}^{p^{m}}(\tau)$. However we have to introduce some $G$ functions (which are not obtained by $S L(2, \mathbf{Z})$ transformation from $G_{0}^{p^{m}}(\tau)$ ), so that we obtain the set of partition functions corresponding to all the types considered in Sec.2.3. 
By using $G_{j}^{p^{m}}(\tau),\left(j=0, \ldots, p^{m}-1\right)$, we introduce the partition function of block $p^{m}$ and type $j$ :

$$
Z_{j}^{p^{m}}(\tau):=\frac{1}{p^{m}}\left(G_{0}^{p^{m}}(\tau)+\zeta_{p^{m}}^{-j} G_{1}^{p^{m}}(\tau)+\cdots+\zeta_{p^{m}}^{-j\left(p^{m}-1\right)} G_{p^{m}-1}^{p^{m}}(\tau)\right), j=0, \ldots, p^{m}-1 .
$$

For the partition function of block $p^{M},(M=m-1, \ldots, 1)$ and type $j$, we introduce

$$
\begin{aligned}
Z_{l p^{M-1}+k}^{p^{M}}(\tau):= & \frac{1}{p^{2 m-M}}\left(G_{0}^{p^{M}}(\tau)+\zeta_{p^{M}}^{-\left(l p^{M-1}+k\right)} G_{1}^{p^{M}}(\tau)+\cdots+\zeta_{p^{M}}^{-\left(l p^{M-1}+k\right)\left(p^{M}-1\right)} G_{p^{M}-1}^{p^{M}}(\tau)\right) \\
& +Z_{k p^{2}}^{p^{M+1}}(\tau), \quad l=0, \ldots, p-1, \quad k=0, \ldots, p^{M-1}-1 .
\end{aligned}
$$

Note that the partition function $Z_{l p^{M-1}+k}^{p^{M}}(\tau)$ consists of $G_{j}^{p^{M}}(\tau),\left(M=0, \ldots, p^{M}-1\right)$, and the partition function $Z_{k p^{2}}^{p^{M+1}}(\tau)$. This is the same reason why the number of orbits of block $p^{l}$ is calculated by the naive number of orbits of block $p^{l}$ and that of block $p^{l-1}$. Finally for the partition function of block 1, we introduce

$$
\begin{aligned}
Z_{t}^{1}(\tau) & :=\frac{1}{p^{2 m}} G_{0}^{1}(\tau)+Z_{0}^{p}(\tau) \\
& =\frac{1}{p^{2 m}} \sum_{\substack{0 \leq a, b, d \in \mathbf{Z} \\
a d=p^{m}, b<d}} d G\left(\frac{a \tau+b}{d}\right) .
\end{aligned}
$$

In the same way as the prime $N$ case, we obtain

$$
\begin{aligned}
Z_{S U\left(p^{m}\right) / \mathbf{Z}_{p}}(\tau):= & \sum_{v_{N}} Z_{v_{N}}(\tau) \\
= & n_{0}^{p^{m}} Z_{0}^{p^{m}}(\tau)+\cdots+n_{p^{m}-1}^{p^{m}} Z_{p^{m}-1}^{p^{m}}(\tau) \\
& +n_{0}^{p^{m-1}} Z_{0}^{p^{m-1}}(\tau)+\cdots+n_{p^{m-1}-1}^{p^{m-1}} Z_{p^{m-1}-1}^{p^{m-1}}(\tau) \\
& +\cdots+n_{0}^{p} Z_{0}^{p}(\tau)+\cdots+n_{p-1}^{p} Z_{p-1}^{p}(\tau)+n_{t}^{1} Z_{t}^{1}(\tau) \\
= & \frac{1}{p^{2 m}} \sum_{\substack{0 \leq a, b, d \in \mathbf{Z} \\
a d=p m, b<d}} d^{12}(\operatorname{gcd}(b, d))^{11} G\left(\frac{a \tau+b}{d}\right) .
\end{aligned}
$$

Last equalities in $(2.49)$ and $(2.50)$ are nothing but the result in 11.

(ii) $N=p q$ case

We introduce the following $G$ functions by $S L(2, \mathbf{Z})$ transformation from $G_{0}^{p q}(\tau)$,

$$
G_{j}^{p q}(\tau):=\left(\frac{1}{\eta\left(\frac{\tau+j}{p q}\right)}\right)^{24}, j=0, \ldots, p q-1 .
$$

Block $p$,

$$
G_{j}^{p}(\tau):=\left(\frac{1}{\eta\left(\frac{q \tau+j}{p}\right)}\right)^{24}, j=0, \ldots, p-1
$$


Block $q$,

$$
G_{j}^{q}(\tau):=\left(\frac{1}{\eta\left(\frac{p \tau+j}{q}\right)}\right)^{24}, j=0, \ldots, q-1,
$$

Block 1,

$$
G_{t}^{1}(\tau):=\left(\frac{1}{\eta(p q \tau)}\right)^{24}
$$

For the partition function of block $p q$, we introduce

$$
Z_{j}^{p q}(\tau):=\frac{1}{p q}\left(G_{0}^{p q}(\tau)+\zeta_{p q}^{-j} G_{1}^{p q}(\tau)+\cdots+\zeta_{p q}^{-j(p q-1)} G_{p q-1}^{p q}(\tau)\right), j=0, \ldots, p q-1,
$$

By imitating the counting of $n_{j}^{p q}$, we introduce the partition function of block $p$,

$$
\begin{aligned}
Z_{l_{k}^{p}}^{p}(\tau):= & \frac{1}{p q^{2}}\left(G_{0}^{p}(\tau)+\zeta_{p}^{-\left(l_{k}^{p}\right)} G_{1}^{p}(\tau)+\cdots+\zeta_{p}^{-\left(l_{k}^{p}\right)(p-1)} G_{p-1}^{p}(\tau)\right) \\
& +Z_{k}^{p q}(\tau), 0 \leq l_{k}^{p} \leq p-1,
\end{aligned}
$$

where $l_{k}^{p}$ satisfies $l_{k}^{p} q^{2} \equiv 2 q k \bmod 2 p q$.

The partition function of block $q$ are similarly introduced.

Finally for block 1, we introduce

$$
\begin{gathered}
Z_{t}^{1}(\tau)=\frac{1}{p^{2} q^{2}} G_{t}^{1}(\tau)+Z_{0}^{p}(\tau)+Z_{0}^{q}(\tau)-Z_{0}^{p q}(\tau) \\
=\frac{1}{p^{2} q^{2}} \sum_{\substack{0 \leq a, b, d \in \mathbf{Z} \\
a d=p q, b<d}} d G\left(\frac{a \tau+b}{d}\right) . \\
Z_{S U(p q) / \mathbf{Z}_{p q}(\tau):=} \sum_{v_{N}} Z_{v_{N}}(\tau) \\
=n_{0}^{p q} Z_{0}^{p q}(\tau)+\cdots+n_{p q-1}^{p^{m}} Z_{p q-1}^{p^{m}}(\tau)+n_{0}^{p} Z_{0}^{p}(\tau)+\cdots+n_{p-1}^{p} Z_{p-1}^{p}(\tau) \\
\quad+n_{0}^{q} Z_{0}^{q}(\tau)+\cdots+n_{q-1}^{q} Z_{q-1}^{q-1}(\tau)+n_{t}^{1} Z_{t}^{1}(\tau) \\
=\frac{1}{p^{2} q^{2}} \sum_{\substack{0 \leq a, b, d \in \mathbf{Z} \\
a d d=p q, b<d}} d^{12}(\operatorname{gcd}(b, d))^{11} G\left(\frac{a \tau+b}{d}\right) .
\end{gathered}
$$

(2.57) and (2.58) are the same as the result in 11.

\section{Stringy Point of View}

In this section, we consider the origin of the duality conjecture on the $\mathcal{N}=4$ Yang-

Mills theory from the stringy point of view. Next, we estimate the general structure of the partition function in the $D, E$ case, based on this observation. In [22], the duality conjecture on gauge theory on $\mathbf{R}^{4}$ is interpreted as the $T$-duality of type II A string theory compactified on $T^{2} \times(A L E$ space). In this context, gauge group is determined by the type of the $A L E$ space, which is classified by the $A D E$ sub-group of $S U(2)$, and the gauge 
coupling is identified with the deformation parameter $\tau$ of the complex structure of $T^{2}$. In this way, $S$-duality transformation of the Yang-Mills theory is derived as the $T$-duality transformation of $T^{2}$.

Now, we apply this interpretation to the $\mathcal{N}=4 A D E$ Yang-Mills theory on $K 3$ surface. We can naturally see that the corresponding string theory is the type II A string theory compactified on $K 3 \times T^{2} \times(A L E$ space). In this case, we have to note that both $K 3$ and $A L E$ space are hyper Kähler surfaces. Therefore, we can choose a surface of which we take zero-volume limit. If we shrink the $T^{2} \times(A L E$ space), we obtain the $\mathcal{N}=4 A D E$ Yang-Mills theory on $K 3$ surface. Then what happens if we shrink $T^{2} \times K 3$ first ? We can speculate naturally that the resulting effective theory is the $\mathcal{N}=4$ gauge theory on $A L E$ space. Then what we have to do next is determination of the gauge group. This can be derived in the following way. Let us assume that $K 3$ surface has the elliptic fibration. Generically, elliptic fibered $K 3$ has 24 singular fibers. Each singular fiber has a simple nodal singularity and generate $U(1)$ gauge symmetry. Therefore, we can see that the resulting gauge group is $U(1)^{24}$. This result is quite natural if we remember the duality between type II A string theory on $K 3$ and heterotic string theory on $T^{4}$ [5]. Since $\mathcal{N}=4$ gauge theory on $K 3$ does not depend on the complex structure of $K 3$, we don't have to consider the gauge symmetry enhancement. In sum, we have obtained the following picture:

$(A D E$ Yang-Mills theory on $K 3) \simeq\left(U(1)^{24}\right.$ Yang-Mills theory on $A D E A L E$ space $)$.

Now, we derive the partition function of $\mathcal{N}=4 U(1)$ gauge theory on the $A L E$ space. We can obtain this theory as a M5-brane world volume theory wrapped on $T^{2} \times(A L E$ space). Appearance of $U(1)$ gauge symmetry corresponds to the case of singly wrapped brane. According to [13], 22], the partition function of singly wrapped M5-brane is determined by the $H^{2}$ lattice and Euler number of the $A L E$ space. Let us denote the $A L E$ space associated with $A D E$ group $\mathcal{G}$ by $A L E(\mathcal{G})$. It is well known that

$$
H^{2}(A L E(\mathcal{G}), \mathbf{Z})=-(\Lambda(\mathcal{G})), \quad \chi(A L E(\mathcal{G}))=r+1,
$$

where $-(\Lambda(\mathcal{G}))$ represents the root lattice whose metric is given by the minus of the standard one. Then the partition function $Z_{U(1)}^{A L E(\mathcal{G})}(\tau)$ is given by

$$
\begin{aligned}
Z_{U(1)}^{A L E(\mathcal{G})}(\tau) & =\frac{\theta_{\mathcal{G}}^{0}(\tau)}{\eta(\tau)^{r+1}}, \\
\theta_{\mathcal{G}}^{0}(\tau) & :=\sum_{m \in \mathbf{Z}^{r}} q^{\frac{1}{2}^{t}(m) C_{\mathcal{G}}(m)} .
\end{aligned}
$$

where $q:=\exp (2 \pi i \tau)$, and $C_{\mathcal{G}}$ is Cartan Matrix of $\mathcal{G}$. Notice that the above result is compatible with the result of Nakajima [19], that asserts that direct sum of the cohomology of the moduli space of $U(1)$ instantons on $A L E(\mathcal{G})$ is decomposed into level 1 representations of the affine Lie algebra of $\mathcal{G}$. Combining (3.1) and (3.3), we estimate (at least as the first approximation) that the partition function $Z_{\mathcal{G}}^{K 3}(\tau)$ of $\mathcal{N}=4 \mathcal{G}$ Yang-Mills theory on $K 3$ surface has the following form:

$$
Z_{\mathcal{G}}^{K 3}(\tau)=\left(\frac{\theta_{\mathcal{G}}^{0}(\tau)}{\eta(\tau)^{r+1}}\right)^{24}
$$




\section{Denominator Identity and Partition Function for $D, E$ Gauge Groups on $K 3$}

In this section, we turn into another suggestive result useful for determination of the partition function of $D, E$ gauge theory. In our previous work [0], we came across a mysterious identity:

$$
\frac{1}{\eta\left(\frac{\tau}{N}\right)}=\frac{\theta_{A_{N-1}}^{2}(\tau)}{\eta(\tau)^{N}}
$$

where $\theta_{A_{N-1}}^{2}(\tau)$ is a kind of $A_{N-1}$ theta function which is explicitly defined for general $A D E$ groups as follows:

Definition 1 For rank $r$ gauge group $\mathcal{G}$, which has dual Coxeter number $h$, half of the sum of the positive roots $\rho$ and roots $\left\{\alpha_{k}\right\}$ (see [8]), we define the following function

$$
\theta_{\mathcal{G}}^{2}(\tau):=\sum_{m \in \mathbf{Z}^{r}} q^{\frac{1}{2}^{t}\left(m+\frac{\rho}{h}\right) C_{\mathcal{G}}\left(m+\frac{\rho}{h}\right)},
$$

where $q:=\exp (2 \pi i \tau)$.

Since the identity (4.1) originates from the celebrated denominator identity of the affine Lie algebra, it is straightforward to generalize (4.1) to $D, E$ case:

Theorem 1 (Macdonald, Kac) [19], 8]

$$
\sum_{m \in \mathbf{Z}^{r}} q^{\frac{1}{2}{ }^{t}\left(m+\frac{\rho}{h}\right) C_{\mathcal{G}}\left(m+\frac{\rho}{h}\right)}=q^{\frac{1}{24} \frac{\operatorname{dim}(\mathcal{G})}{h}} \prod_{n=1}^{\infty}\left(1-q^{\frac{n}{h}}\right)^{r} \prod_{\alpha_{k}}\left(1-q^{\frac{n}{h}} \zeta_{h}^{<\rho, \alpha_{k}>}\right),
$$

where $\zeta_{h}:=\exp (2 \pi i / h)$.

Furthermore as will be shown in appendix B, the r.h.s. of (4.3) can be expressed as $\eta$ product.

With the consideration in the previous section, we had better regard this identity as the one that interpolate the previous estimation of the partition function in (3.4) to the well-known form given by $\eta$-product. Of course, we have to note that the theta function appearing in $(4.3)$ is not $\theta_{\mathcal{G}}^{0}(\tau)$ but $\theta_{\mathcal{G}}^{2}(\tau)$. This subtle translation by $\frac{\rho}{h}$ in the theta sum is puzzling, and seems to be caused by boundary condition of the $A L E$ space. This point should be pursued further, but instead, we consider here how to use the identity (4.3). To this aim, we look back at the construction of $A_{N}$ partition function given in Section 2. In Section 2, we used $G_{0}(\tau)=\left(\theta_{A_{N-1}}^{2}(\tau) / \eta(\tau)^{N}\right)^{24}$ as the primary building block and summed up the $S L(2, \mathbf{Z})$ orbit of $\left(\theta_{A_{N-1}}^{2}(\tau) / \eta(\tau)^{N}\right)^{24}$ so that the whole partition functions satisfy the duality conjecture. Therefore, we expect that the identity (4.3) tells us how to modify the first approximation (3.4) of the $D, E$ partition function on $K 3$. The reason why the $A_{N}$ partition function is written in the form of $\eta$-product comes from the fact that the moduli space of $S U(N)$ instanton on $K 3$ is described by Hilbert scheme of points on $K 3$. This fact can be derived by Fourier-Mukai transform of semi-stable sheaves on $K 3$. Since we treat $D, E$ type instanton on the same $K 3$, we speculate that some analogous structure may exist in the moduli space of $D, E$ type instanton on $K 3$. In other words, the 
primary building block of the $D, E$ partition function should take the form of $\eta$-product. Here, we show the table of the $\eta$-products obtained from the identity (4.3). With these consideration, we choose for the primary function of $D, E$ gauge groups,

$$
G_{0}(\tau)=\left(\frac{\theta_{\mathcal{G}}^{2}(\tau)}{\eta(\tau)^{r+1}}\right)^{24} .
$$

Because these functions have the form of $\eta$-product, we can repeat the same process in Sec.2.4 for these primary functions. As a result, we obtain the partition function for $D, E$ gauge groups on $K 3$, which satisfy Montonen-Olive duality (2.8). Though our computation stems from a wild speculation, it is quite non-trivial that the resulting partition functions satisfy Montonen-Olive duality condition in all the cases we test.

Before moving to the individual case, we will sketch the processes of the determination of $D, E$ partition function on $K 3$. First we have to prepare the sets of $G$ functions. Here we point out that each $D, E$ theory has the corresponding $S U(N)$ theory characterized by its center. We prepare $G$ functions so that all the $G$ functions reproduce the corresponding $S U(N)$ structure. Most of $G$ 's are obtained by $S L(2, \mathbf{Z})$ transformation of $G_{0}(\tau)$, but some $G$ functions are added in order to satisfy the above condition. Basically one can obtain $D, E$ partition function on $K 3$ by imposing Montonen-Olive duality on some combination of $G$ functions. However we also introduce the intermediate functions $\tilde{G}$, which are some combination of $G$ functions. Here $S L(2, \mathbf{Z})$ transformation of $\tilde{G}$ functions is the same as that of $G$ functions of the corresponding $S U(N)$ theory.

\section{1 $D_{2 N}$}

$D_{2 N}$ is dual to $D_{2 N} / \mathbf{Z}_{2} \times \mathbf{Z}_{2}$. Thus we introduce 't Hooft flux $v \in H^{2}\left(K 3, \mathbf{Z}_{2} \times \mathbf{Z}_{2}\right)$. As we have already saw in Sec.2.3, $Z_{v}(\tau)$ are classified by degenerated $\mathbf{Z}_{2}$. In this subsection, we try to derive $D_{2}$ and $D_{4}$ explicitly.

$D_{2}$

Following the $S U(2)$ case, we first introduce the $G$ functions obtained from transforming $G_{0}(\tau)$ by $S L(2, \mathbf{Z})$.

$$
\begin{gathered}
G_{j}(\tau):=\left(\frac{\eta(\tau)}{\eta\left(\frac{\tau+j}{2}\right)^{2}}\right)^{24}, j=0,1 \\
H_{0}(\tau):=\left(\frac{\eta(\tau)}{\eta(2 \tau)^{2}}\right)^{24} .
\end{gathered}
$$

To define the partition function for even type $\left(v^{2} \equiv 0\right)$ and odd type $\left(v^{2} \equiv 2\right)$, we introduce

$$
Z_{j}(\tau):=\frac{1}{4}\left(G_{0}(\tau)+(-1)^{j} G_{1}(\tau)\right), j=0,1,
$$

We also introduce the partition function of trivial type,

$$
Z_{t}(\tau):=\frac{1}{16} H_{0}(\tau)+Z_{0}(\tau)
$$

By using (4.7) and (4.8) and the numbers $\left\{n_{j}\right\}$ in Sec.2.3, we obtain

$$
Z_{D_{2} / \mathbf{Z}_{2} \times \mathbf{Z}_{2}}(\tau):=n_{0} Z_{0}(\tau)+n_{1} Z_{1}(\tau)+n_{t} Z_{t}(\tau) .
$$


(4.8) and (4.9) surely satisfy Montonen-Olive duality (2.8),

$$
Z_{t}\left(-\frac{1}{\tau}\right)=4^{-11}\left(\frac{\tau}{i}\right)^{-12} Z_{D_{2} / \mathbf{Z}_{2} \times \mathbf{Z}_{2}}(\tau)
$$

Note that the coefficients of $G$ 's in (4.7) and (4.8) are determined by imposing (4.10).

$$
D_{4}
$$

Following the $D_{2}$ case, we first introduce the $G$ functions obtained from transforming $G_{0}(\tau)$ by $S L(2, \mathbf{Z})$.

$$
\begin{aligned}
G_{j}(\tau) & :=\left(\frac{\eta\left(\frac{\tau+j}{3}\right)}{\eta\left(\frac{\tau+j}{2}\right) \eta\left(\frac{\tau+j}{6}\right)}\right)^{24}, j=0, \ldots, 5, \\
H_{j}(\tau) & :=\left(\frac{\eta\left(\frac{\tau+j}{3}\right)}{\eta(2 \tau) \eta\left(\frac{2 \tau+2 j}{3}\right)}\right)^{24}, j=0, \ldots, 2, \\
I_{j}(\tau) & :=\left(\frac{\eta(3 \tau)}{\eta\left(\frac{\tau+j}{2}\right) \eta\left(\frac{3 \tau+3 j}{2}\right)}\right)^{24}, j=0,1, \\
& J_{0}(\tau):=\left(\frac{\eta(3 \tau)}{\eta(2 \tau) \eta(6 \tau)}\right)^{24} .
\end{aligned}
$$

Contrary to the $D_{2}$ case, we will introduce the intermediate $\tilde{G}(\tau)$ functions, so that we pick up the powers $q^{(1 / 2) \mathbf{Z}}$ from the $G$ functions having $q^{(1 / 6) \mathbf{Z}}$ powers,

$$
\begin{gathered}
\tilde{G}_{j}(\tau):=G_{j}(\tau)+G_{2+j}(\tau)+G_{4+j}(\tau)+I_{j}(\tau), j=0,1 \\
\tilde{H}_{0}(\tau):=H_{0}(\tau)+H_{1}(\tau)+H_{2}(\tau)+J_{0}(\tau)
\end{gathered}
$$

Once we introduce $\tilde{G}(\tau)$ 's, we only follow the same processes as $D_{2}$ case. Actually the modular property of $\tilde{G}_{j}(\tau)$ and $\tilde{H}_{0}(\tau)$ is completely the same as that of $G_{j}(\tau)$ and $H_{0}(\tau)$ in $D_{2}$.

$$
\begin{gathered}
Z_{j}(\tau):=\frac{1}{4}\left(\tilde{G}_{0}(\tau)+(-1)^{j} \tilde{G}_{1}(\tau)\right), j=0,1, \\
Z_{t}(\tau):=\frac{1}{16} \tilde{H}_{0}(\tau)+Z_{0}(\tau), \\
Z_{D_{4} / \mathbf{Z}_{2} \times \mathbf{Z}_{2}}(\tau):=n_{0} Z_{0}(\tau)+n_{1} Z_{1}(\tau)+n_{t} Z_{t}(\tau),
\end{gathered}
$$

(4.18) and (4.19) also satisfy Montonen-Olive duality (2.8),

$$
Z_{t}\left(-\frac{1}{\tau}\right)=4^{-11}\left(\frac{\tau}{i}\right)^{-12} Z_{D_{4} / \mathbf{Z}_{2} \times \mathbf{Z}_{2}}(\tau) .
$$

\section{$4.2 \quad D_{2 N+1}$}

$D_{2 N+1}$ is dual to $D_{2 N+1} / \mathbf{Z}_{4}$. Thus we introduce 't Hooft flux $v \in H^{2}\left(K 3, \mathbf{Z}_{4}\right)$. As we have already saw in Sec.2.3, $Z_{v}(\tau)$ are classified by $\mathbf{Z}_{4}$. This structure is the same as $A_{3}$ case. Indeed

$$
D_{3}=A_{3}
$$

In this subsection, we try to derive the $D_{5}$ partition function explicitly. 
$D_{5}$

Following the $S U(4)$ case, we first introduce the $G$ functions obtained from transforming $G_{0}(\tau)$ by $S L(2, \mathbf{Z})$, and add some $G$ functions so that all $G$ functions form the modular property of $S U(4)$.

$$
\begin{gathered}
G_{j}(\tau):=\left(\frac{\eta\left(\frac{\tau+j}{4}\right)}{\eta\left(\frac{\tau+j}{2}\right) \eta\left(\frac{\tau+j}{8}\right)}\right)^{24}, j=0, \ldots, 7, \\
H_{j}(\tau):=\left(\frac{\eta\left(\frac{2 \tau+j}{2}\right)}{\eta(2 \tau) \eta\left(\frac{2 \tau+j}{4}\right)}\right)^{24}, j=0, \ldots, 3, \\
I_{0}(\tau):=\left(\frac{\eta(4 \tau)}{\eta(2 \tau) \eta\left(\frac{4 \tau+1}{2}\right)}\right)^{24}, \\
J_{0}(\tau):=\left(\frac{\eta(4 \tau)}{\eta(2 \tau) \eta(8 \tau)}\right)^{24}, \\
K_{j}(\tau):=\left(\frac{\eta(2 \tau)}{\eta\left(\frac{2 \tau+j}{2}\right)^{2}}\right)^{24}, j=0,1, \\
L_{j}(\tau):=\left(\frac{\eta\left(\frac{\tau+j}{2}\right)}{\eta\left(\frac{\tau+j}{4}\right)^{2}}\right)^{24}, j=0, \ldots, 3, \\
M_{0}(\tau):=\left(\frac{\eta(2 \tau)}{\eta(4 \tau)^{2}}\right)^{24} .
\end{gathered}
$$

Precisely speaking, $G_{j},(j=0, \ldots, 7), H_{1}, H_{3}, I_{0}, J_{0}$ are obtained from transforming $G_{0}(\tau)$ by $S L(2, \mathbf{Z})$, and $H_{0}, H_{2}, K_{j},(j=0,1), L_{j},(j=0, \ldots, 3), M_{0}$ are obtained from requiring the modular property of $S U(4)$.

For the same reason as the $D_{4}$ case, we will introduce the intermediate $\tilde{G}(\tau)$ functions, so that we pick up the powers $q^{(1 / 4) \mathbf{Z}}$ from $G$ functions,

$$
\begin{gathered}
\tilde{G}_{j}^{4}(\tau):=G_{j}(\tau)+G_{4+j}(\tau)+2^{12} L_{j}(\tau), j=0, \ldots, 3 \\
\tilde{G}_{j}^{2}(\tau):=H_{j}(\tau)+H_{2+j}(\tau)+2^{12} K_{j}, j=0,1, \\
\tilde{G}_{t}^{1}(\tau):=2^{12} I_{0}(\tau)+J_{0}(\tau)+M_{0}(\tau) .
\end{gathered}
$$

The modular property of $\tilde{G}_{j}^{4}(\tau), \tilde{G}_{j}^{2}(\tau)$ and $\tilde{G}_{t}^{1}(\tau)$ is completely the same as that of $G_{j}^{4}(\tau)$, $G_{j}^{2}(\tau)$ and $G_{t}^{1}(\tau)$ in $S U(4)$ respectively. Mysterious $2^{12}$ factor in (4.28), (4.29) and (4.30) is needed for this equivalence. By using $\tilde{G}(\tau)$ 's, we follow the same processes as the $S U(4)$ case.

$$
\begin{gathered}
Z_{j}^{4}(\tau):=\frac{1}{4}\left(\tilde{G}_{0}^{4}(\tau)+\zeta_{4}^{-j} \tilde{G}_{1}^{4}(\tau)+\cdots+\zeta_{4}^{-j 3} \tilde{G}_{3}^{1}(\tau)\right), j=0, \ldots, 3, \\
Z_{j}^{2}(\tau):=\frac{1}{8}\left(\tilde{G}_{0}^{2}(\tau)+(-1)^{j} \tilde{G}_{1}^{2}(\tau)\right)+Z_{0}^{4}(\tau), j=0,1, \\
Z_{t}^{1}(\tau):=\frac{1}{16} \tilde{G}_{t}^{1}(\tau)+Z_{0}^{2}(\tau), \\
Z_{D_{5} / \mathbf{Z}_{4}}(\tau):=n_{0}^{4} Z_{0}^{4}(\tau)+\cdots+n_{3}^{4} Z_{N-1}^{4}(\tau)+n_{0}^{2} Z_{0}^{2}(\tau)+n_{1}^{2} Z_{1}^{2}(\tau)+n_{t}^{1} Z_{t}^{1}(\tau) .
\end{gathered}
$$


(4.33) and (4.34) indeed satisfy Montonen-Olive duality (2.8) in the same way as $S U(4)$ case,

$$
Z_{t}^{1}\left(-\frac{1}{\tau}\right)=4^{-11}\left(\frac{\tau}{i}\right)^{-12} Z_{D_{5} / \mathbf{Z}_{4}}(\tau)
$$

\section{$4.3 E_{6,7,8}$}

$E_{6}$

$E_{6}$ is dual to $E_{6} / \mathbf{Z}_{3}$. Thus we introduce 't Hooft flux $v \in H^{2}\left(K 3, \mathbf{Z}_{3}\right)$. As we already saw in Sec.2.3, $Z_{v}(\tau)$ are classified by $\mathbf{Z}_{3}$. Explicit $G$ functions are written in Appendix C. Following the $D_{4}$ and $D_{5}$ case, we introduce the intermediate functions,

$$
\begin{gathered}
\tilde{G}_{j}(\tau):=\sum_{k=0}^{3} G_{3 k+j}(\tau)+\sum_{k=0}^{1} H_{3 k+3-j}(\tau)+\sum_{k=0}^{1} N_{3 k+j}(\tau)+J_{3-j}(\tau), j=0,1,2, \\
\tilde{H}_{0}(\tau):=\sum_{k=0}^{3} I_{k}(\tau)+\sum_{k=0}^{1} K_{k}(\tau)+\sum_{k=0}^{1} M_{k}(\tau)+L_{0}(\tau) .
\end{gathered}
$$

By using $\tilde{G}(\tau)$ 's, we follow the same process as the $S U(3)$ case.

$$
\begin{gathered}
Z_{j}(\tau):=\frac{1}{3}\left(\tilde{G}_{0}(\tau)+\zeta_{3}^{-j} \tilde{G}_{1}(\tau)+\zeta_{3}^{-j 2} \tilde{G}_{2}(\tau)\right), j=0,1,2 \\
Z_{t}(\tau):=\frac{1}{9} \tilde{H}_{0}(\tau)+Z_{0}(\tau) \\
Z_{E_{6} / \mathbf{Z}_{3}}(\tau):=n_{0} Z_{0}(\tau)+n_{1} Z_{1}(\tau)+n_{2} Z_{2}(\tau)+n_{t} Z_{t}(\tau) .
\end{gathered}
$$

(4.39) and (4.40) surely satisfy Montonen-Olive duality (2.8) in the same way as the $S U(3)$ case,

$$
Z_{t}\left(-\frac{1}{\tau}\right)=3^{-11}\left(\frac{\tau}{i}\right)^{-12} Z_{E_{6} / \mathbf{Z}_{3}}(\tau)
$$

$E_{7}$

By repeating the similar processes to $E_{6}$ case, the results of $E_{7}$ are given by

$$
\begin{gathered}
\tilde{G}_{j}(\tau):=\sum_{k=0}^{8} G_{2 k+j}(\tau)+\sum_{k=0}^{2} I_{2 k+j}(\tau)+\sum_{k=0}^{2} N_{2 k+j}(\tau)+K_{j}(\tau), j=0,1 \\
\tilde{H}_{0}(\tau):=\sum_{k=0}^{8} H_{k}(\tau)+\sum_{k=0}^{3} J_{k}(\tau)+\sum_{k=0}^{2} M_{k}(\tau)+L_{0}(\tau)
\end{gathered}
$$

Corresponding $S U(N)$ theory is $S U(2)$. Thus we define

$$
\begin{gathered}
Z_{j}(\tau):=\frac{1}{2}\left(\tilde{G}_{0}(\tau)+(-1)^{-j} \tilde{G}_{1}(\tau)\right), j=0,1 \\
Z_{t}(\tau):=\frac{1}{4} \tilde{H}_{0}(\tau)+Z_{0}(\tau) \\
Z_{E_{7} / \mathbf{Z}_{2}}(\tau):=n_{0} Z_{0}(\tau)+n_{1} Z_{1}(\tau)+n_{t} Z_{t}(\tau)
\end{gathered}
$$


(4.45) and (4.46) satisfy Montonen-Olive duality (2.8) in the same way as the $S U(2)$ case,

$$
Z_{t}\left(-\frac{1}{\tau}\right)=2^{-11}\left(\frac{\tau}{i}\right)^{-12} Z_{E_{7} / \mathbf{Z}_{2}}(\tau)
$$

$E_{8}$

Since $E_{8}$ is self-dual group, $Z_{t}(\tau)$ turns out to be a modular form of weight -12 . In this case, we introduce $G$ functions obtained from transforming $G_{0}$ by $S L(2, \mathbf{Z})$.

$$
\begin{aligned}
G_{j}(\tau) & :=\left(\frac{\eta\left(\frac{\tau+j}{6}\right) \eta\left(\frac{\tau+j}{10}\right) \eta\left(\frac{\tau+j}{15}\right)}{\eta\left(\frac{\tau+j}{2}\right) \eta\left(\frac{\tau+j}{3}\right) \eta\left(\frac{\tau+j}{5}\right) \eta\left(\frac{\tau+j}{30}\right)}\right)^{24}, j=0, \ldots, 29, \\
H_{j}(\tau) & :=\left(\frac{\eta\left(\frac{2 \tau+2 j}{3}\right) \eta\left(\frac{2 \tau+2 j}{5}\right) \eta\left(\frac{\tau+j}{15}\right)}{\eta(2 \tau) \eta\left(\frac{\tau+j}{3}\right) \eta\left(\frac{\tau+j}{5}\right) \eta\left(\frac{2 \tau+2 j}{15}\right)}\right)^{24}, j=0, \ldots, 14, \\
I_{j}(\tau) & :=\left(\frac{\eta\left(\frac{3 \tau+3 j}{2}\right) \eta\left(\frac{\tau+j}{10}\right) \eta\left(\frac{3 \tau+3 j}{5}\right)}{\eta\left(\frac{\tau+j}{2}\right) \eta(3 \tau) \eta\left(\frac{\tau+j}{5}\right) \eta\left(\frac{3 \tau+3 j}{10}\right)}\right)^{24}, j=0, \ldots, 9, \\
J_{j}(\tau) & :=\left(\frac{\eta\left(\frac{\tau+j}{6}\right) \eta\left(\frac{5 \tau+5 j}{2}\right) \eta\left(\frac{5 \tau+5 j}{3}\right)}{\eta\left(\frac{\tau+j}{2}\right) \eta\left(\frac{\tau+j}{3}\right) \eta(5 \tau) \eta\left(\frac{5 \tau+5 j}{6}\right)}\right)^{24}, j=0, \ldots, 5,5,4, \\
K_{j}(\tau) & :=\left(\frac{\eta(6 \tau) \eta\left(\frac{2 \tau+2 j}{5}\right) \eta\left(\frac{3 \tau+3 j}{5}\right)}{\eta(2 \tau) \eta(3 \tau) \eta\left(\frac{\tau+j}{5}\right) \eta\left(\frac{6 \tau+6 j}{5}\right)}\right)^{24}, j=0, \ldots, 4,2, \\
L_{j}(\tau) & :=\left(\frac{\eta\left(\frac{2 \tau+2 j}{3}\right) \eta(10 \tau) \eta\left(\frac{5 \tau+5 j}{3}\right)}{\eta(2 \tau) \eta\left(\frac{\tau+j}{3}\right) \eta(5 \tau) \eta\left(\frac{10 \tau+10 j}{3}\right)}\right)^{24}, j=0,1,2, \\
M_{j}(\tau) & :=\left(\frac{\eta\left(\frac{3 \tau+j}{2}\right) \eta\left(\frac{5 \tau+j}{2}\right) \eta(15 \tau)}{\eta\left(\frac{\tau+j}{2}\right) \eta(3 \tau) \eta(5 \tau) \eta\left(\frac{15 \tau+j}{2}\right)}\right)^{24}, j=0,1, \\
& N_{0}(\tau):=\left(\frac{\eta(6 \tau) \eta(10 \tau) \eta(15 \tau)}{\eta(2 \tau) \eta(3 \tau) \eta(5 \tau) \eta(30 \tau)}\right)
\end{aligned}
$$

Each $G$ function is transformed to each other by $S L(2, \mathbf{Z})$ with no extra factor except for $\left(\frac{\tau}{i}\right)^{-12}$. Therefore, there is no need to introduce the intermediate functions. The partition function is simply given by adding all the $G$ functions.

$$
\begin{aligned}
Z_{t}(\tau):= & \sum_{j=0}^{29} G_{j}(\tau)+\sum_{j=0}^{14} H_{j}(\tau)+\sum_{j=0}^{9} I_{j}(\tau)+\sum_{j=0}^{5} J_{j}(\tau)+\sum_{j=0}^{4} K_{j}(\tau)+\sum_{j=0}^{2} L_{j}(\tau) \\
& +\sum_{j=0}^{1} M_{j}(\tau)+N_{0}(\tau) .
\end{aligned}
$$

Therefore, (4.56) is surely self-dual or a modular form,

$$
Z_{t}\left(-\frac{1}{\tau}\right)=\left(\frac{\tau}{i}\right)^{-12} Z_{t}(\tau)
$$


Indeed $Z_{t}(\tau)$ can be expressed by the well-known modular forms as follows:

$$
\begin{aligned}
Z_{t}(\tau)= & \frac{1}{\eta(\tau)^{24}}\left(j^{8}(\tau)-5976 j^{7}(\tau)+14049204 j^{6}(\tau)-16450492296 j^{5}(\tau)\right. \\
& +10006744823442 j^{4}(\tau)-2995100782701144 j^{3}(\tau)+373127947258066100 j^{2}(\tau) \\
& -12808385327808647208 j(\tau)+26763599994092029512),
\end{aligned}
$$

where $j(\tau)$ is the famous modular $j$-function [14]. Notice that

$$
j(\tau)=\chi_{1,0}(\tau)^{3}
$$

where $\chi_{1,0}(\tau)$ is level one $E_{8}$ character. In this way, $Z_{t}(\tau)$ can be expressed only in terms of $\chi_{1,0}(\tau)$ and $\eta(\tau)$. This fact ensures that $Z_{t}(\tau)$ is a good candidate for the $E_{8}$ partition function on $K 3$.

\section{$5 \quad$ Holomorphic Anomaly ?}

In this section, we discuss that our partition function for $D, E$ gauge group is not sufficient from the point of view of the gap condition, and search for the improvement analogous to the holomorphic anomaly on $\frac{1}{2} K 3[13]$.

First, we derive the gap condition for $A D E$ gauge groups by using the famous AtiyahHitchin-Singer dimension formula for the moduli space of irreducible anti-self-dual connections with $A D E$ gauge group $\mathcal{G}$ on $X[1]$.

$$
\operatorname{dim} \mathcal{M}_{k}^{\mathcal{G}}=4 h(\mathcal{G}) k-\operatorname{dim} \mathcal{G} \frac{\chi(X)+\sigma(X)}{2},
$$

where $h(\mathcal{G})$ is the dual Coxeter number, $\operatorname{dim} \mathcal{G}$ is the dimension of $\mathcal{G}$ and $k$ is the instanton number. $\chi(X)$ and $\sigma(X)$ are Euler number and signature of $X$ respectively. In our case of $X=K 3$, this formula reduces to

$$
\operatorname{dim} \mathcal{M}_{k}^{\mathcal{G}}=4 h(\mathcal{G}) k-4 \operatorname{dim} \mathcal{G} .
$$

Here we point out that $h(\mathcal{G}), \operatorname{dim}(\mathcal{G})$ and $r$ are related by the following formula:

$$
\operatorname{dim}(\mathcal{G})=r h(\mathcal{G})+r, h(\mathcal{G})>r .
$$

Therefore,

$$
\operatorname{dim} \mathcal{M}_{k}^{\mathcal{G}}=4 h(\mathcal{G})(k-r)-4 r .
$$

$\operatorname{dim} \mathcal{M}_{k}^{\mathcal{G}} \geq 0$ means $k \geq r+1$. That is, the moduli space of irreducible ASD connections with $A D E$ gauge group on $K 3$ can exist only for $k \geq r+1$. This condition restricts the form of the partition function. Remember that the partition function has the form:

$$
Z_{v}^{X}(\tau):=q^{-\frac{(r+1) \chi(X)}{24}} \sum_{k} \chi(\mathcal{M}(v, k)) q^{k}
$$

In our case, we consider the case of $X=K 3$ and of trivial flux, which corresponds to $\mathcal{M}(0, k)=\mathcal{M}_{k}^{\mathcal{G}}$. Thus we obtain

$$
Z_{t}(\tau)=q^{-(r+1)} \sum_{k} \chi\left(\mathcal{M}_{k}^{\mathcal{G}}\right) q^{k}
$$


This form and the condition $k \geq r+1$ requires (5.6) to start from $\mathcal{O}\left(q^{0}\right)$ term. Furthermore, it is well-known that the trivial connection always exists [21]. This means that the contribution from $k=0$ appears in (5.6) for any gauge group. Therefore, forbidden $q$ powers in (5.6) are $q^{-r}, \ldots, q^{-1}$, that is $1 \leq k \leq r$. This is the gap condition.

The $S U(N)$ partition function on $K 3$ surely satisfies this gap condition [21]. However our $D, E$ partition function on $K 3$ does not satisfy this gap condition. To discuss this problem, we will pick up the $E_{8}$ partition function on $K 3$. The other $D, E$ partition functions on $K 3$ have the same problem as the $E_{8}$ case. First we expand $Z_{t}(\tau)$ of $E_{8}$ :

$$
\begin{aligned}
Z_{t}(\tau)= & q^{-9}+24 q^{-7}+24 q^{-6}+324 q^{-5}+600 q^{-4}+3500 q^{-3}+10008 q^{-2}+22890627 q^{-1} \\
& +134931884553792+1697352144366449440512 q \\
& +177293857723945037797591920 q^{2}+2444259891832216559852811815364 q^{3} \\
& +10159099239429369126457917291280224 q^{4}+\cdots .
\end{aligned}
$$

What is the origin of $q^{-7}, q^{-6}, \ldots, q^{-1}$ ? We can suggest the following two possibilities. The one possibility is the failure of the vanishing theorem [21]. The vanishing theorem ensures the equality (5.6). From this view point, we may have derived the $D, E$ partition function on $K 3$, which corresponds to the generating function of the Euler number of the generalized moduli space including the field $A_{\mu}, B_{\mu \nu}^{+}$and $C$ [21, 10]. The second possibility is the appearance of the reducible connection. Atiyah-Hitchin-Singer dimension formula is the formula for irreducible ASD connection. Thus if there are reducible connections, it is no wonder why the negative powers appear in the partition function. Actually the above two possibilities are mutually related. We will discuss the second possibility more in detail here.

First we point out the well-known fact that there is a holomorphic anomaly in the $S U(N)$ partition function on the manifold $b_{2}^{+} \leq 1$. This holomorphic anomaly comes from reducible connections. We think that our situation is very similar to the holomorphic anomaly appearing in the $U(N)$ partition function on $\frac{1}{2} K 3$ [13, 3]. For example, let us expand the holomorphic part of the $U(2)$ partition function $Z_{2}^{\text {hol. }}(\tau)$, which does not include $E_{2}(\tau)$ terms [13, 3].

$$
Z_{2}^{\text {hol. }}(\tau)=\frac{1}{12} q^{-1}-20-11787 q-\frac{2132320}{3} q^{2}+\cdots .
$$

If one adds $E_{2}(\tau)$ terms, which are determined by the holomorphic anomaly equation [13], one can obtain $Z_{2}(\tau)$, which includes no $\mathcal{O}\left(q^{0}\right)$ term. Looking back at our problem, we may obtain the complete partition function of $D, E$ gauge theory by adding $E_{2}(\tau)$ dependent terms, which come from reducible connections. Since $E_{2}(\tau)$ behave anomalously under $S L(2, \mathbf{Z})$ transformation, each coefficient function should be a modular form of appropriate weight. More explicitly, expected full partition function $Z_{t}^{f u l l}(\tau)$ should take the following form:

$$
\begin{aligned}
Z_{t}^{\text {full }}(\tau) & =\frac{1}{\eta(\tau)^{216}} \cdot\left(\sum_{j}\left(E_{2}(\tau)\right)^{j} \cdot f_{j}\left(E_{4}, E_{6}\right)\right) \\
Z_{t}(\tau) & =\frac{f_{0}\left(E_{4}, E_{6}\right)}{\eta(\tau)^{216}}
\end{aligned}
$$


where $f_{j}\left(E_{4}, E_{6}\right)$ is a modular form of weight $96-2 j$. Of course, the above ansatz has enough degrees of freedom to cancel the coefficient of $q^{-7}, q^{-6}, \ldots, q^{-1}$ of $Z_{t}(\tau)$. But we don't have the principle to select the "right" $f_{j}\left(E_{4}, E_{6}\right)$ up to now. We expect to find the analogue of holomorphic anomaly equation used in [13]. Unfortunately, corresponding Seiberg-Witten curve is not known and the problem still needs further consideration.

\section{Conclusion and Discussion}

We have determined the holomorphic part of the $D, E$ partition function on $K 3$, by using the $D, E$ blow-up formula. This partition function completely satisfies Montonen-Olive duality.

Remaining problem is determination of the complete partition function of $D, E$ gauge theory on $K 3$, which satisfies the gap condition. We think that the desired complete $D, E$ partition function on $K 3$ includes the holomorphic part and the anti-holomorphic part having $E_{2}(\tau)$ terms. We also expect that the anti-holomorphic part of the partition function of $D, E$ gauge theory on $K 3$ should be determined by some equations, which corresponds to the holomorphic anomaly equation on $\frac{1}{2} K 3$. To this aim, an approach from Seiberg-Witten curve seems to be effective since the holomorphic anomaly equation was verified from the analysis Seiberg-Witten of $\mathcal{N}=4$ gauge theory.

Acknowledgment

We would like to thank T.Eguchi, K.Fukaya, H.Nakajima, K.Ono and K.Yoshioka for helpful suggestions and useful discussions. We owe the major part of Sec.5 to the discussions with them. 


\section{A Modular property of $S U(3) / \mathbf{Z}_{3}$ theory}

In this article, we only considered the modular property of (2.8).

$$
Z_{t}\left(-\frac{1}{\tau}\right)=3^{-11} \tau^{-12}\left(n_{0} Z_{0}(\tau)+n_{1} Z_{1}(\tau)+n_{2} Z_{2}(\tau)+n_{t} Z_{t}(\tau)\right)
$$

Of course other modular properties in (2.6) must be considered. In this section, we derive other modular properties in (2.6) by counting the orbits for $A_{2}$ case.

\section{A.1 $Z_{0}\left(-\frac{1}{\tau}\right)$}

$$
Z_{0}\left(-\frac{1}{\tau}\right)=3^{-11} \tau^{-12}\left(m_{0} Z_{0}(\tau)+m_{1} Z_{1}(\tau)+m_{2} Z_{2}(\tau)+m_{t} Z_{t}(\tau)\right) .
$$

We determine $\left\{m_{j}\right\}$ by using (2.6).

$$
\left\{\begin{array}{c}
m_{0}=m_{0}^{0}+\zeta_{3} m_{0}^{1}+\zeta_{3}^{2} m_{0}^{2} \\
m_{1}=m_{1}^{0}+\zeta_{3} m_{1}^{1}+\zeta_{3}^{2} m_{1}^{2} \\
m_{2}=m_{2}^{0}+\zeta_{3} m_{2}^{1}+\zeta_{3}^{2} m_{2}^{2} \\
m_{t}=m_{t}^{0}
\end{array}\right.
$$

where

$$
\begin{gathered}
m_{j}^{k}=\text { number of } u^{2} \equiv 2 j \bmod 6 \text { and } V_{1} \cdot u \equiv k \bmod 3, u \neq 0, \\
m_{t}^{0}=1 .
\end{gathered}
$$

Here $V_{1}^{2}=0$ and $V_{1} \neq 0$,and $v=V_{1}$ corresponds to $v^{2} \equiv 0 \bmod 6$ and $v \neq 0$. The results are given by

$$
\begin{aligned}
& \left\{\begin{array}{c}
m_{0}^{0}=3^{20}+2 \cdot 3^{10}-1 \\
m_{0}^{1}=3^{20} \\
m_{0}^{2}=3^{20} \\
m_{0}=2 \cdot 3^{10}-1
\end{array}\right. \\
& \left\{\begin{array}{c}
m_{1}^{0}=3^{20}-3^{10} \\
m_{1}^{1}=3^{20} \\
m_{1}^{2}=3^{20} \\
m_{1}=-3^{10}
\end{array}\right. \\
& \left\{\begin{array}{c}
m_{2}^{0}=3^{20}-3^{10} \\
m_{2}^{1}=3^{20} \\
m_{2}^{2}=3^{20} \\
m_{2}=-3^{10}
\end{array}\right.
\end{aligned}
$$

A.2 $Z_{1}\left(-\frac{1}{\tau}\right)$

$$
Z_{1}\left(-\frac{1}{\tau}\right)=3^{-11} \tau^{-12}\left(m_{0} Z_{0}(\tau)+m_{1} Z_{1}(\tau)+m_{2} Z_{2}(\tau)+m_{t} Z_{t}(\tau)\right) .
$$

We determine $\left\{m_{j}\right\}$ by using (2.6). 


$$
\left\{\begin{array}{c}
m_{0}=m_{0}^{0}+\zeta_{3} m_{0}^{1}+\zeta_{3}^{2} m_{0}^{2} \\
m_{1}=m_{1}^{0}+\zeta_{3} m_{1}^{1}+\zeta_{3}^{2} m_{1}^{2} \\
m_{2}=m_{2}^{0}+\zeta_{3} m_{2}^{1}+\zeta_{3}^{2} m_{2}^{2} \\
m_{t}=m_{t}^{0}
\end{array}\right.
$$

where

$$
\begin{gathered}
m_{j}^{k}=\text { number of } u^{2} \equiv 2 j \bmod 6 \text { and }\left(V_{1}+V_{2}\right) \cdot u \equiv k \bmod 3, u \neq 0, \\
m_{t}^{0}=1 .
\end{gathered}
$$

Here $\left(V_{1}+V_{2}\right)^{2}=2$, and $v=V_{1}+V_{2}$ corresponds to $v^{2} \equiv 2 \bmod 6$. The results are given by

$$
\begin{gathered}
\left\{\begin{array}{c}
m_{0}^{0}=3^{20}-1 \\
m_{0}^{1}=3^{20}+3^{10} \\
m_{0}^{2}=3^{20}+3^{10} \\
m_{0}=-3^{10}-1
\end{array}\right. \\
\left\{\begin{array}{c}
m_{1}^{0}=3^{20}-3^{10} \\
m_{1}^{1}=3^{20} \\
m_{1}^{2}=3^{20} \\
m_{1}=-3^{10}
\end{array}\right. \\
\left\{\begin{array}{c}
m_{2}^{0}=3^{20}+3^{10} \\
m_{2}^{1}=3^{20}-3^{10} \\
m_{2}^{2}=3^{20}-3^{10} \\
m_{2}=2 \cdot 3^{10}
\end{array}\right.
\end{gathered}
$$

\section{B $A D E$ Blow-up formula}

\section{B.1 $A_{r}$ case}

It is convenient to write roots $\alpha_{k}$ of $A_{r}$ algebra by orthonormal basis $\left\{e_{j}\right\}$. We write $\rho$ by these basis. By using these data, we derive $\theta_{A_{r}}^{2}(\tau)$ as below.

$$
\begin{gathered}
\operatorname{Roots}\left\{\alpha_{k}\right\}: \quad e_{i}-e_{j}, \quad(1 \leq i \neq j \leq r+1) . \\
h=r+1, \\
\rho=\frac{1}{2}\left(r e_{1}+(r-2 \cdot 1) e_{2}+\cdots+(r-2 \cdot r) e_{r+1}\right), \\
\zeta_{h}=\exp \left(\frac{2 \pi i}{h}\right), \\
\theta_{A_{r}}^{2}(\tau)=q^{\frac{1}{24} \frac{r(r+2)}{h}} \prod_{n=1}^{\infty}\left(1-q^{\frac{n}{h}}\right)^{r} \prod_{\alpha_{k}}\left(1-q^{\frac{n}{h}} \zeta_{h}^{<\rho, \alpha_{k}>}\right) \\
=\frac{\eta(\tau)^{r+1}}{\eta\left(\frac{\tau}{h}\right)}, \\
G_{0}(\tau):=\left(\frac{\theta_{A_{r}}^{2}(\tau)}{\eta(\tau)^{r+1}}\right)^{24}=\left(\frac{1}{\eta\left(\frac{\tau}{h}\right)}\right)^{24} .
\end{gathered}
$$




\section{B.2 $D_{r}$ case}

$$
\begin{gathered}
\operatorname{Roots}\left\{\alpha_{k}\right\}: \pm e_{i} \pm e_{j}, \quad(1 \leq i \neq j \leq r) . \\
h=2 r-2, \\
\rho=\frac{1}{2}\left(h e_{1}+(h-2) e_{2}+\cdots+2 e_{r-1}\right), \\
\zeta_{h}=\exp \left(\frac{2 \pi i}{h}\right), \\
\theta_{D_{r}}^{2}(\tau)=q^{\frac{1}{24} \frac{r(2 r-1)}{h}} \prod_{n=1}^{\infty}\left(1-q^{\frac{n}{h}}\right)^{r} \prod_{\alpha_{k}}\left(1-q^{\frac{n}{h}} \zeta_{h}^{<\rho, \alpha_{k}>}\right) \\
=\frac{\eta(\tau)^{r+1} \eta\left(\frac{2 \tau}{h}\right)}{\eta\left(\frac{\tau}{2}\right) \eta\left(\frac{\tau}{h}\right)}, \\
G_{0}(\tau):=\left(\frac{\theta_{D_{r}}^{2}(\tau)}{\eta(\tau)^{r+1}}\right)^{24}=\left(\frac{\eta\left(\frac{2 \tau}{h}\right)}{\eta\left(\frac{\tau}{2}\right) \eta\left(\frac{\tau}{h}\right)}\right)^{24} .
\end{gathered}
$$

\section{B.3 $E_{6}$ case}

$$
\begin{gathered}
\text { Roots }\left\{\alpha_{k}\right\}: \pm e_{i} \pm e_{j} \quad(1 \leq i \neq j \leq 5), \\
\frac{1}{2}\left( \pm e_{1} \pm e_{2} \cdots \pm \sqrt{3} e_{6}\right), \text { even }+ \text { 's. } \\
h=12, \\
\rho=\frac{1}{2}\left(16 e_{1}+6 e_{2}+4 e_{3}+2 e_{4}\right), \\
\zeta_{12}=\exp \left(\frac{2 \pi i}{12}\right), \\
\theta_{E_{6}}^{2}(\tau)=q^{\frac{1}{24} \frac{78}{12}} \prod_{n=1}^{\infty}\left(1-q^{\frac{n}{h}}\right)^{6} \prod_{\alpha_{k}}\left(1-q^{\frac{n}{h}} \zeta_{h}^{<\rho, \alpha_{k}>}\right) \\
=\frac{\eta(\tau)^{7} \eta\left(\frac{\tau}{4}\right) \eta\left(\frac{\tau}{6}\right)}{\eta\left(\frac{\tau}{2}\right) \eta\left(\frac{\tau}{3}\right) \eta\left(\frac{\tau}{12}\right)}, \\
G_{0}(\tau):=\left(\frac{\eta\left(\frac{\tau}{4}\right) \eta\left(\frac{\tau}{6}\right)}{\eta\left(\frac{\tau}{2}\right) \eta\left(\frac{\tau}{3}\right) \eta\left(\frac{\tau}{12}\right)}\right)^{24} .
\end{gathered}
$$




\section{B.4 $E_{7}$ case}

$$
\begin{gathered}
\text { Roots }\left\{\alpha_{k}\right\}: \pm e_{i} \pm e_{j} \quad(1 \leq i \neq j \leq 6), \quad \pm \sqrt{2} e_{7} \\
\frac{1}{2}\left( \pm e_{1} \pm e_{2} \cdots \pm \sqrt{3} e_{7}\right), \quad \text { even }+ \text { 's. } \\
h=18, \\
\rho=\frac{1}{2}\left(26 e_{1}+8 e_{2}+6 e_{3}+4 e_{4}+2 e_{5}+\sqrt{2} e_{7}\right), \\
\zeta_{18}=\exp \left(\frac{2 \pi i}{18}\right), \\
\theta_{E_{7}}^{2}(\tau)=q^{\frac{1}{24} \frac{133}{18}} \prod_{n=1}^{\infty}\left(1-q^{\frac{n}{h}}\right)^{7} \prod_{\alpha_{k}}\left(1-q^{\frac{n}{h}} \zeta_{h}^{<\rho, \alpha_{k}>}\right) \\
=\frac{\eta(\tau)^{8} \eta\left(\frac{\tau}{6}\right) \eta\left(\frac{\tau}{9}\right)}{\eta\left(\frac{\tau}{2}\right) \eta\left(\frac{\tau}{3}\right) \eta\left(\frac{\tau}{18}\right)}, \\
G_{0}(\tau):=\left(\frac{\eta\left(\frac{\tau}{6}\right) \eta\left(\frac{\tau}{9}\right)}{\eta\left(\frac{\tau}{2}\right) \eta\left(\frac{\tau}{3}\right) \eta\left(\frac{\tau}{18}\right)}\right)^{24} .
\end{gathered}
$$

\section{B.5 $E_{8}$ case}

$$
\begin{gathered}
\operatorname{Roots}\left\{\alpha_{k}\right\}: \pm e_{i} \pm e_{j} \quad(1 \leq i \neq j \leq 8), \\
\frac{1}{2}\left( \pm e_{1} \pm e_{2} \cdots \pm e_{8}\right), \quad \text { even }+ \text { 's. } \\
h=30, \\
\rho=\frac{1}{2}\left(46 e_{1}+12 e_{2}+10 e_{3} \cdots+2 e_{7}\right), \\
\zeta_{30}=\exp \left(\frac{2 \pi i}{30}\right), \\
\theta_{E_{8}}^{2}(\tau)=\frac{q^{\frac{1}{24}} \frac{248}{30} \prod_{n=1}^{\infty}\left(1-q^{\frac{n}{h}}\right)^{8} \prod_{\alpha_{k}}\left(1-q^{\frac{n}{h}} \zeta_{h}^{<\rho, \alpha_{k}>}\right)}{\eta(\tau)^{9} \eta\left(\frac{\tau}{6}\right) \eta\left(\frac{\tau}{10}\right) \eta\left(\frac{\tau}{15}\right)} \\
G_{0}(\tau) \eta\left(\frac{\tau}{3}\right) \eta\left(\frac{\tau}{5}\right) \eta\left(\frac{\tau}{30}\right)
\end{gathered}
$$




\section{C $\quad G$ functions of $E_{6}$ and $E_{7}$}

\section{C.1 Functions of $E_{6}$}

We introduce $G$ functions given by $S L(2, \mathbf{Z})$ transformation from $G_{0}(\tau)$. Furthermore we add some $G$ functions, so that all $G$ functions satisfy the modular properties of $S U(3)$.

$$
\begin{aligned}
G_{j}(\tau) & :=\left(\frac{\eta\left(\frac{\tau+j}{4}\right) \eta\left(\frac{\tau+j}{6}\right)}{\eta\left(\frac{\tau+j}{2}\right) \eta\left(\frac{\tau+j}{3}\right) \eta\left(\frac{\tau+j}{12}\right)}\right)^{24}, j=0, \ldots, 11, \\
H_{j}(\tau) & :=\left(\frac{\eta\left(\frac{2 \tau+j}{2}\right) \eta\left(\frac{2 \tau+j}{3}\right)}{\eta(2 \tau) \eta\left(\frac{\tau-j}{3}\right) \eta\left(\frac{2 \tau+j}{6}\right)}\right)^{24}, j=0, \ldots, 6, \\
I_{j}(\tau) & :=\left(\frac{\eta\left(\frac{\tau-j}{4}\right) \eta\left(\frac{3 \tau+j}{2}\right)}{\eta\left(\frac{\tau+j}{2}\right) \eta(3 \tau) \eta\left(\frac{3 \tau+j}{4}\right)}\right)^{24}, j=0, \ldots, 3, \\
J_{j}(\tau) & :=\left(\frac{\eta(4 \tau) \eta\left(\frac{2 \tau+j}{3}\right)}{\eta(2 \tau) \eta\left(\frac{\tau-j}{3}\right) \eta\left(\frac{4 \tau+2 j}{3}\right)}\right)^{24}, j=0, \ldots, 2, \\
K_{j}(\tau) & :=\left(\frac{\eta\left(\frac{2 \tau+j}{2}\right) \eta(6 \tau)}{\eta(2 \tau) \eta(3 \tau) \eta\left(\frac{6 \tau+j}{2}\right)}\right)^{24}, j=0,1, \\
L_{0}(\tau):=\left(\frac{\eta(4 \tau) \eta(6 \tau)}{\eta(2 \tau) \eta(3 \tau) \eta(12 \tau)}\right) & , \\
M_{j}(\tau) & :=\left(\frac{\eta(\tau) \eta\left(\frac{3 \tau+3 j}{2}\right)}{\eta\left(\frac{\tau+j}{2}\right) \eta(3 \tau) \eta(3 \tau)}\right)^{24}, j=0,1, \\
N_{j}(\tau) & :=\left(\frac{\eta(\tau) \eta\left(\frac{\tau+j}{6}\right)}{\eta\left(\frac{\tau+j}{2}\right) \eta\left(\frac{\tau+j}{3}\right) \eta\left(\frac{\tau+j}{3}\right)}\right)^{24}, j=0, \ldots, 5 .
\end{aligned}
$$

To obtain $G_{j}(\tau), \ldots, L_{0}(\tau)$, we have to take account of the structure of order 12 Hecke

transformation. $M_{j}(\tau)$ and $N_{j}(\tau)$ are obtained from $S L(2, \mathbf{Z})$ transformation of these functions.

\section{C.2 Function of $E_{7}$}

$$
\begin{aligned}
G_{j}(\tau) & :=\left(\frac{\eta\left(\frac{\tau+j}{6}\right) \eta\left(\frac{\tau+j}{9}\right)}{\eta\left(\frac{\tau+j}{2}\right) \eta\left(\frac{\tau+j}{3}\right) \eta\left(\frac{\tau+j}{18}\right)}\right)^{24}, j=0, \ldots, 17, \\
H_{j}(\tau) & :=\left(\frac{\eta\left(\frac{2 \tau+2 j}{3}\right) \eta\left(\frac{\tau+j}{9}\right)}{\eta(2 \tau) \eta\left(\frac{\tau+j}{3}\right) \eta\left(\frac{2 \tau+2 j}{9}\right)}\right)^{24}, j=0, \ldots, 8, \\
I_{j}(\tau) & :=\left(\frac{\eta\left(\frac{3 \tau+j}{2}\right) \eta\left(\frac{3 \tau+j}{3}\right)}{\eta\left(\frac{\tau+j}{2}\right) \eta(3 \tau) \eta\left(\frac{3 \tau+j}{6}\right)}\right)^{24}, j=0, \ldots, 5, \\
J_{j}(\tau) & :=\left(\frac{\eta(6 \tau) \eta\left(\frac{3 \tau+j}{3}\right)}{\eta(2 \tau) \eta(3 \tau) \eta\left(\frac{6 \tau+2 j}{3}\right)}\right)^{24}, j=0, \ldots, 2,
\end{aligned}
$$




$$
\begin{gathered}
K_{j}(\tau):=\left(\frac{\eta\left(\frac{3 \tau+j}{2}\right) \eta(9 \tau)}{\eta\left(\frac{\tau+j}{2}\right) \eta(3 \tau) \eta\left(\frac{9 \tau+j}{2}\right)}\right)^{24}, j=0,1, \\
L_{0}(\tau):=\left(\frac{\eta(6 \tau) \eta(9 \tau)}{\eta(2 \tau) \eta(3 \tau) \eta(18 \tau)}\right)^{24}, \\
M_{j}(\tau):=\left(\frac{\eta(\tau) \eta\left(\frac{2 \tau+2 j}{3}\right)}{\eta(2 \tau) \eta(2 \tau) \eta\left(\frac{\tau+j}{3}\right)}\right)^{24}, j=0,1,2, \\
N_{j}(\tau):=\left(\frac{\eta(\tau) \eta\left(\frac{\tau+j}{6}\right)}{\eta\left(\frac{\tau+j}{2}\right) \eta\left(\frac{\tau+j}{2}\right) \eta\left(\frac{\tau+j}{3}\right)}\right)^{24}, j=0, \ldots, 5 .
\end{gathered}
$$

\section{References}

[1] M.F.Atiyah, N.H.Hitchin and I.M.Singer. Self-duality in four dimensional Riemannian Geometry, Proc.R.Soc.Lond A362(1978)425.

[2] G.Bonelli. The geometry of M5-branes and TQFTs, hep-th/0012075.

[3] T.Eguchi and K.Sakai. Seiberg-Witten Curve for the E-String Theory, hep-th/0203025.

[4] K.Fukaya. Topology, geometry and field theory, World Scientific 1994.

[5] J. A. Harvey, A. Strominger. The Heterotic String is a Soliton, Nucl.Phys. B449 (1995) $535-552$

[6] M.Jinzenji and T.Sasaki. $N=4$ Supersymmetric Yang-Mills Theory on Orbifold$T^{4} / \mathbf{Z}_{\mathbf{2}}$, Mod.Phys.Lett. A16 (2001) 411-428.

[7] M.Jinzenji and T.Sasaki. $N=4$ Supersymmetric Yang-Mills Theory on Orbifold$T^{4} / \mathbf{Z}_{2}$ :Higher Rank Case, JHEP 0112 (2001) 002.

[8] V. G. Kac. Infinite dimensional Lie algebras, Cambridge 1990.

[9] M.Kapranov. The Elliptic Curve in the S-Duality Theory and Eisenstein Series for Kac-Moody Groups, math.AG/0001005.

[10] J. M. F. Labastida, Carlos Lozano. Mathai-Quillen formulation of twisted $\mathcal{N}=4$ supersymmetric gauge theories in four dimensions, Nucl.Phys.B502(1997)741.

[11] J. M. F. Labastida, Carlos Lozano. The Vafa-Witten Theory for Gauge Group SU(N) hep-th/9903172.

[12] I.G.Macdonald. Affine root systems and Dedekind's $\eta$-functions, Invent. math. 15 (1972), 91-143.

[13] J. A. Minahan, D. Nemeschansky, C. Vafa, N. P. Warner. E-Strings and N=4 Topological Yang-Mills Theories Nucl.Phys. B 527 (1998) 581-623.

[14] T.Miyake. Modular Forms New York,Springer-Verlag(1989). 
[15] T. Nakanishi, A. Tsuchiya. Level-Rank Duality of WZW Models in Conformal Field Theory, Commun. Math. Phys. 144, 351-372 (1992).

[16] C.Montonen and D.Olive. Phys.Lett.B 72 (1977) 117;

P.Goddard, J.Nyuts and D.Olive. Nucl. Phys. B125 (1977) 1.

[17] S.Mukai. Inv.Math. 77 (1984) 101;

L.Göttsche. Math.Ann. 286 (1990) 193.

[18] H.Nakajima. Lectures on Hilbert schemes of points on surfaces, to appear.

[19] H.Nakajima. Instantons on ALE spaces, quiver varieties, and Kac-Moody algebras, Duke Math. J. 76 (1994);

Gauge theory on resolutions of simple singularities and simple Lie algebras., Internat. Math. Res. Notices 1994.

[20] G.’t Hooft. , Nucl.Phys.B138(1978)1;Nucl.Phys.B153(1979)141.

[21] C.Vafa and E.Witten. A strong coupling test of S-duality, Nucl. Phys. B431 (1994) 3.

[22] C.Vafa. Geometric Origin of Montonen-Olive Duality, Adv.Theor.Math.Phys. 1 (1998) 158-166.

[23] E.Witten. Supersymmetric Yang-Mills Theory on A Four Manifold. J. Math. Phys. 35 (1994) 5101-5135.

[24] K.Yoshioka. The Betti numbers of the moduli space of stable sheaves of rank 2 on $\mathbf{P}^{2}$. J. reine angew. Math. 453 (1994), 193-220.

[25] K.Yoshioka. Euler characteristics of SU(2) instanton moduli spaces on rational elliptic surfaces Commun.Math.Phys. 205 (1999) 501-517.

[26] K.Yoshioka. Irreducibility of moduli spaces of vector bundles on K3 surfaces math.AG/9907001.

[27] K.Yoshioka. Betti numbers of the moduli space of stable sheaves on some surfaces, Nucl.Phys.B (Proc.Suppl.) 46 (1996) 263-268. 


\begin{tabular}{|c|c|}
\hline $\mathcal{G}$ & $\theta_{\mathcal{G}}^{2}(\tau) / \eta(\tau)^{r+1}$ \\
\hline$A_{r}$ & $\frac{1}{\eta\left(\frac{\tau}{r+1}\right)}$ \\
\hline$D_{r}$ & $\frac{\eta\left(\frac{\tau}{r-1}\right)}{\eta\left(\frac{\tau}{2}\right) \eta\left(\frac{\tau}{2 r-2}\right)}$ \\
\hline$E_{6}$ & $\frac{\eta\left(\frac{\tau}{4}\right) \eta\left(\frac{\tau}{6}\right)}{\eta\left(\frac{\tau}{2}\right) \eta\left(\frac{\tau}{3}\right) \eta\left(\frac{\tau}{12}\right)}$ \\
\hline$E_{7}$ & $\frac{\eta\left(\frac{\tau}{6}\right) \eta\left(\frac{\tau}{9}\right)}{\eta\left(\frac{\tau}{2}\right) \eta\left(\frac{\tau}{3}\right) \eta\left(\frac{\tau}{18}\right)}$ \\
\hline$E_{8}$ & $\frac{\eta\left(\frac{\tau}{6}\right) \eta\left(\frac{\tau}{10}\right) \eta\left(\frac{\tau}{15}\right)}{\eta\left(\frac{\tau}{2}\right) \eta\left(\frac{\tau}{3}\right) \eta\left(\frac{\tau}{5}\right) \eta\left(\frac{\tau}{30}\right)}$ \\
\hline
\end{tabular}

